\title{
VERDI E IL WAGNERISMO NEL MOVIMENTO GERMANICO ITALIANO
}

\author{
ANTONIO ROSTAGNO (*)
}

\begin{abstract}
RIASSUNTO - "Via prussiana" e "liberalismo classico" sono due concetti opposti; nella distanza che li separa si colloca la preoccupazione di Verdi per il "germanesimo". Verdi incontra la cultura tedesca molto precocemente: nel 1834 dirige La creazione di Haydn e nel 1838 pone in musica due traduzioni dal Faust di Goethe. Ma in questo momento Goethe e Haydn sono considerati cultura universale, non esempi di "germanesimo". La cultura tedesca sarà invece sentita come entità estranea, come potenziale colonizzatrice, solo con l'emergere del nazionalismo prussiano, dopo Sedan. Tutto è cambiato, e Verdi protesta con un teatrale "ora tutto è tedesco"; ma ora al posto di Haydn c'è Wagner; al posto di Goethe c'è Heine. Ciò che preoccupa Verdi non è tanto un fatto artistico, quanto un pericolo più generale, radicale e penetrante. L'Italia infatti con governi di Francesco Crispi si sta avviando su una nuova "via prussiana", un "movimento tedesco", che è anzitutto politico ed economico, prima che artistico. Al raffreddamento dei rapporti con la Francia (e la "guerra doganale"), consegue l'avvicinamento al modello-Bismarck. La Triplice Alleanza, in questo senso, è la matrice del wagnerismo italiano; e lo sgomento di Verdi deriva non dalla situazione artistica (o meglio, non solo), ma da questa onda che sommerge tutti gli aspetti della vita, mettendo in pericolo il processo di costruzione nazionale. Verdi è uno dei primi a comprendere quanto la "via prussiana" sia profondamente inadatta a una nazione nata sui fondamenti etici, prima che politici, del liberalismo classico.
\end{abstract}

$* * *$

ABSTRACT - Deutsche bewegung (Prussian way) and "classical liberalism" are two opposite concepts; and in the gap between them Verdi's concern about the "germanesimo" is to be placed. Verdi meets the German culture very early: in 1834 he conducts Haydn's Die Schöpfung and in 1838 writes two songs on Goethe's Faust Italian translations. But at that time Goethe and Haydn are considered as universal culture and not as particular "german" expressions. German culture will be seen as an foreign entity, as a potential colonizer, only with the rise of Prussian nationalism, after French débâcle at

(*) Università di Roma "La Sapienza". 
Sedan. Then everything changes, and Verdi raises his voice complaining that "now all is German"; but now in Haydn's place there is Wagner; in the place of Goethe there is Heine. What worries Verdi is not only the artistic situation, but a more general, radical and deep danger. Actually under the government of Francesco Crispi Italy is starting on a new "Prussian way", e Deutsche bewegung, a "movement" more political and economic, than artistical. The approaching to the Bismarck model is a consequence of the worsening of relationship between Italy and France (until the so-called Guerra doganale, "Tariff war". The Triplice Alleanza is so one of the main origins of Italian Wagnerism; and Verdi's dismay doesn't follow from the artistic situation (or better, not only), but from the real wave which submerged the whole everyday life, endangering the process of national consciousness. Verdi is one of the first who understood to what extent the "Prussian way" was unsuitable to a nation grounded on classic liberalism ethical, before than political principles.

Il wagnerismo italiano è un fenomeno assai complesso, che va ben oltre il campo musicale, e che manifesta pulsioni profonde della psicologia collettiva della giovane nazione. Di conseguenza anche la relazione fra Verdi e il wagnerismo va collegata alle dinamiche politiche, all'opinione pubblica e ai comportamenti sociali di quel momento. È ciò che proverò a fare.

Sostenere che il significato di Aida si risolva nel problema del potere politico esercitato dalla casta sacerdotale sarebbe un'eccessiva semplificazione, se non proprio un errore; affermare che Simon Boccanegra è un capolavoro perché tematizza lo scontro di classe e la denuncia di autonomismi regionali sarebbe ugualmente insensato, sebbene quei temi siano presenti nella versione 1881. Il valore estetico e la qualità drammaturgica non si collocano su questo piano; una lettura in chiave storico-politica non può cogliere che parziali aspetti di queste grandi opere verdiane. Ma di lì a concludere che le relazioni fra l'opera e la storia politica siano solo elementi contingenti del melodramma di Verdi, che costituiscano solo la parte materiale senza incidenza sulla parte estetica, che appartengano al piano dell'accidentale e non del sostanziale dell'opera d'arte, è un sillogismo di cui comprendo l'intenzione, ma che non riesco ad accettare.

Anticipo subito un esempio in cui la relazione fra il piano della storia della cultura e il piano della drammaturgia apporta una determinazione essenziale. La qualità tragica di Otello deriva da una particolare posizione di Verdi nel contesto culturale e politico del 1887, proviene dalla personale percezione di estraneità al presente, di tardività e di anacronismo che Verdi prova in questo momento; tale modo di perce- 
pirsi si traduce, sublimato, nel contenuto estetico dell'opera. Sono anni dominati dalla cultura positivista nei vari suoi campi di applicazione, non ultime la psicologia e la pedagogia. Lombroso, Mantegazza, Gabelli, Villari, Morselli, Ferri, Sergi, Roncoroni, Ferrero, Bianchi ed altri danno il loro apporto (chi più chi meno) alle nuove discipline della psicologia sperimentale e della psicologia sociale. A partire da Lombroso gli elementi comportamentali della devianza criminale sono posti in relazione a fattori scientifici, ereditari, statistici: in tal modo $i$ nuovi psicologi arrivano a definire il crimine, il male, come una conseguenza statisticamente dedotta da dati anatomico-biologici e da fattori ambientali. La colpa e la responsabilità del delitto sono sottratte al soggetto, al libero arbitrio, a fattori di scelta individuale. Anche il criterio della punizione, quindi, è misurato sull'incidenza del fattore ambientale, togliendo responsabilità all'individuo (e quindi imputabilità: in tal modo il piano scientifico ha immediate ricadute sul piano giuridico, e di qui al piano politico il passo è breve). Alla punizione si tende a sostituire la comprensione, per enfatizzare la funzione costrittiva, deterministica che l'individuo subisce dal condizionamento biologico-ambientale. Anche la letteratura segue questo indirizzo con romanzi come Giacinta di Capuana (1879) e soprattutto con il dilagare del "romanzo sperimentale" da Zola, anche nelle trasposizioni sceniche di diverse compagnie italiane. ${ }^{1}$ Francesco De Sanctis è uno dei più acuti interpreti di questa situazione:

Avevamo l'umanismo, oggi abbiamo l'animalismo nella sua esagerazione. È chiaro che in questo nuovo ambiente c'è qualcosa di basso e di corrotto, che si vuol purificato. E ciò avverrà, ove il nostro spirito sia disposto a guardare l'uomo meno nelle somiglianze già assorbite, e più nelle differenze, che gli danno il diritto di dire: - Sono un uomo e non un animale - . $^{2}$

1 Zacconi, Novelli, la Pezzana, Giovani Emanuel, Adelaide Tessero, la Duse ed altri introducono nel teatro italiano molti titoli zoliani, dalla Teresa Raquin all'Assommoir, il dramma simbolo della nuova corrente (cfr. Roberto Alonge, Teatro e spettacolo nel secondo Ottocento, Roma-Bari, Laterza, 1988, in particolare il cap. IV "Mattatori, trafficanti ed esattori delle tasse"; Armando Petrini, Attori e scena nel teatro italiano di fine Ottocento. Studio critico su Giovanni Emanuel e Giacinta Pezzana, Torino, Università degli Studi di Torino, 2002).

2 Francesco De Sanctis, Il darwinismo nell'arte, conferenza tenuta al Roma l'11 marzo 1883, ora in Saggi critici, a cura di L. Russo, Bari, Laterza, 1957, vol. III. 
Verdi, come De Sanctis, non può accettare questa spersonalizzazione della realtà, questa emorragia di responsabilità individuale, la sua formazione e la sua convinzione liberale pongono sempre al centro il soggetto inteso come persona, non come oggetto di studio statistico; i suoi personaggi negativi non sono passiva espressione di colpe maturate nelle condizioni sociali, i cui comportamenti sono giustificabili per via di deduzione scientifica-sperimentale. Consideriamo l'antagonista creato da Verdi in questi anni Ottanta, Jago: è un personaggio a cui non si adatta nulla della "teoria dell'uomo delinquente" di Lombroso, ${ }^{3}$ non la fisiognomica, non la giustificazione deterministico-ambientale della "degenerazione" (parola frequentissima nella psicologia sperimentale di questo momento). Arriverei a dire che Jago può essere letto come la denuncia dell'inutilità di tutti questi sforzi scientifici positivisti; è il simbolo del male essenziale dell'uomo, sottratto a condizioni ambientali e a reazioni individuali determinate dall'ereditarietà. Il male non è, nel suo celebre "Credo blasfemo" dell'Atto II, frutto di natura, di disposizioni biologiche, anatomiche; ma ancor meno è determinato dall'ambiente; e meno ancora è studiabile (e curabile) attraverso statistiche dei tratti somatici. È significativo, a questo proposito, il carteggio con cui Verdi chiede a Domenico Morelli di fargli un bozzetto del suo personaggio (quando ancora crede di intitolare l'opera Jago, appunto), dove viene contraddetta implicitamente tutta la dottrina frenologica di Lombroso. Nulla di deviante, delinquenziale o "degenerato" deve trasparire dalla sua fisionomia, ma all'opposto deve esser elegante e seducente, non deve avere nessuno dei tratti del criminale che Lombroso indica nelle sue statistiche:

Bene, benone, benissimo, benississssimo! Jago colla faccia da galantuomo! Hai colpito! [...] Mi par di vederlo questo prete, cioè questo Jago colla faccia da uomo giusto! ${ }^{4}$

pp. 355-367: 367. Riflessioni altrettanto utili al nostro obiettivo si trovano anche nei suoi Studio sopra Emilio Zola (in "Roma” 1878, ora in Saggi cit., pp. 266-312) e Zola e l' Assommoir" (conferenza al Circolo Filosofico di Napoli 15 giugno 1879, ora ivi, pp. 313-337).

3 Cesare Lombroso, L'uomo delinquente in rapporto all' antropologia, alla medicina legale ed alle discipline carcerarie, Milano, Hoepli, 1876.

4 Lettera di Verdi al pittore Domenico Morelli, Genova, 7 febbraio 1880 [ma 1881] (Copialettere, p. 693). 
Verdi in tal modo denuncia (implicitamente, non s'intende dire che questa fosse sua intenzione consapevole) che la devianza, la corruzione, il vizio, il negativo radicale non è affatto annidato nelle terre del brigantaggio, nelle classi più emarginate, nelle categorie esteriormente abbruttite, nella plebe, nel sotto-proletariato, ma è proprio nei più raffinati dandies (come Verdi voleva Jago ritratto da Morelli, appunto). ${ }^{5}$ Sono i dandies decadenti che popolano l'attuale corte bizantina, ${ }^{6}$ ossia la "terza Roma", sotto la cui apparente eleganza e raffinatezza si nasconde il gioco alla distruzione morale del prossimo. Nel 1885 Fogazzaro aveva descritto nel Daniele Cortis questo ambiente; più tardi e ancor più violentemente vi tornerà Pirandello con I vecchi e $i$ giovani. E proprio qui sta la profonda disillusione di Verdi, che certo non può credere nelle prospettive di progresso e di risanamento morale proposte dal positivismo (d'altronde poco dopo nuovamente liquidate dal neo-idealismo primo-novecentesco). Per antifrasi, quindi, Jago diventa una aperta critica alle certezze del positivismo e della psicologia sociale di fine Ottocento.

Questo è un caso, a mio avviso molto significativo, di intreccio fra piano contestuale e piano estetico-drammaturgico; e questa prospettiva, credo, permette una diversa lettura del wagnerismo italiano e della posizione di Verdi. Né ciò dipende da una volontà d'autore, da una tendenza individuale di Verdi, ma da un movimento collettivo, comune; dagli anni Quaranta si era infatti avviato un crescente e sempre più diffuso interesse per le discussioni politiche, per la definizione dei concetti di liberalismo, patria, nazione, stato, costituzionalismo, repubblicanesimo, per la funzione sociale della chiesa ecc. La consapevolezza politica si amplia a più larghi strati sociali nella nuova Italia borghese; il

5 Verdi descrive anche il comportamento che immagina per Jago nella lettera a Morelli del 24 settembre 1881: "di fare distratto, nonchalant, indifferente a tutto, incredulo, frizzante, dicendo il bene e il male con leggerezza come avendo l'aria di pensare a tutt'altro" (Copialettere p. 317).

6 L'aggettivo fa riferimento alla celebre testata La cronaca bizantina, pubblicata a Roma dall'editore Angelo Sommaruga a partire dai primi anni Ottanta, alla quale collaborano numerosi intellettuali del momento fra cui il giovane D'Annunzio. Il titolo del periodico fa esplicito riferimento all'Ode in memoria del patriota Vincenzo Caldesi, in cui Carducci nel 1871 denuncia precocemente la "degenerazione" del governo romano: "Dormi, povero morto. Ancor la soma/ ci grava del peccato:/ impronta Italia domandava Roma,/ Bisanzio essi le han dato". 
discorso della politica coinvolge strati sociali, che tradizionalmente non discorrevano di questi argomenti. I meridionalisti, la Sinistra storica, la Consorteria, la Nuova scuola, la Pentarchia, ecc. non sono precisamente partiti moderni, ma hanno la funzione di far prendere coscienza, di far parteggiare, di indurre una scelta di parte in un gran parte dei cittadini della Nuova Italia; inizia un moderno dibattito politico largamente diffuso. Questa fu un'importante conseguenza di un movimento che era iniziato sin dagli scritti di Vincenzo Gioberti (Il primato degli Italiani, 1843) e Cesare Balbo (Le speranze d'Italia, 1844), ${ }^{7}$ ossia esattamente con la generazione di Verdi, e in larga parte dal coinvolgimento che il mazzinianesimo aveva indotto verso categorie sociali fin'allora estranee a questi discorsi (operai, donne, giovani):

Si trattava di libri [quelli di Gioberti, Balbo] che portarono a parlare di politica e di nazione italiana anche uomini e ambienti che fino ad allora erano rimasti piuttosto estranei alla politica: "se mai la nostra lingua ha avuto bisogno di una nuova parola [politicare] per esprimere una nuova cosa [l'avvento della politica] affé che questo è il caso". ${ }^{8}$

Se questo vale per gli anni intorno al 1848, molto più diviene valido nella nuova Italia borghese, soprattutto con l'allargamento del suffragio avviato dalla Sinistra Storica dal 1876. E fra questi "ambienti [un tempo] estranei alla politica" era anche quello del melodramma; ma con Verdi questa "estraneità" finisce, più per atteggiamento generazionale collettivo, che per sua esplicita intenzione: il contesto di cui Gioberti, Balbo, Guerrazzi, Montanelli, Giusti, Nievo, Gustavo Modena, Settembrini e De Sanctis (solo per nominarne alcuni fra anni Quaranta e Sessanta) sono testimoni è lo stesso nel quale si collocano le

7 Sia chiaro che non suggerisco neppur lontanamente un legame fra le discussioni teoriche di Gioberti e il movimento di allargamento dell'interesse politico a fine secolo; semplicemente quei due libri, con Le mie prigioni di Pellico, Le confessioni di un italiano di Nievo, ovviamente Manzoni e pochi altri hanno ottenuto una diffusione talmente vasta che hanno funzionato come traino per un più capillare interesse per questioni politiche, almeno per quelle più semplici e fondamentali.

8 Antonio Chiavistelli, Moderati/Democratici in Atlante culturale del Risorgimento. Lessico del linguaggio politico dal Settecento all'Unità, Roma-Bari, Laterza, 2011, p. 127; le virgolette indicano una citazione da un commento di Filippo Ugolini (1855) agli effetti del Primato di Gioberti. 
opere di Verdi. Al di là delle intenzioni dell'autore, quindi, questo nuovo contesto genera un tipo di melodramma che non nasce nelle stesse condizioni del precedente; e questo è un contenuto essenziale (ossia connaturato sin dall'origine) del dramma verdiano; che Verdi lo volesse o no, che noi lo vogliamo o no. Anche al di là di intenzionali contenuti politici, quindi, la mentalità di cui il melodramma di Verdi era espressione, l'uditorio a cui si rivolgeva, avrebbe comunque interpretato i messaggi in una chiave politica, in linea con il clima del momento; siamo proprio certi che simili contenuti non debbano essere considerati parte del contenuto sostanziale di quei melodrammi?

E quanto più Verdi acquista consapevolezza del suo ruolo sociale e politico, tanto più tali connessioni vanno indagate a fondo. Gli anni Settanta-Ottanta diventano, in questa prospettiva, un campo di indagine assai ampio, dove le possibili intersezioni fra melodramma verdiano e coeva letteratura politica italiana portano a interessanti conclusioni, ben oltre i luoghi comuni (Verdi patriottico, padre della patria, maestro della rivoluzione, ${ }^{9}$ e via di questo passo ...). In appendice allego una Tabella cronologica che può forse apparire semplificante, ma permette maggiore concretezza; prima di proseguire la lettura sarebbe utile una sua attenta consultazione.

In sintesi, ecco alcune coppie di opposti che definiscono il contesto negli anni successivi a Roma capitale, delineando il campo d'opinione pubblica in cui Verdi compone le ultime opere: individualismo e collettivismo, idealismo e positivismo, pessimismo decadente e ottimismo progressista e attivo, ${ }^{10}$ antiparlamentarismo e centralità dell'ammi-

9 Sintagma quest'ultimo tanto diffuso a cavaliere dei due secoli, quanto inutile e strumentale: intendo non tanto il fatto che Verdi sia effettivamente stato il testimone-cantore di quel momento, quanto il fatto che il Risorgimento non fu una rivoluzione. E quindi quel sintagma va posto di pari passo con i discorsi parlamentari di Francesco Crispi, che ingannava i suoi avversari di sinistra (per portarli dalla sua parte) sostenendo che il suo governo apertamente autoritario e accentratore era in realtà la diretta conseguenza dello spirito rivoluzionario degli anni Quaranta: è la reazione che si copre con la maschera della rivoluzione, persuadendo i vecchi compagni.

10 Forse è giusto anche parlare di pragmatismo, se non fosse qualità comune anche all'idealismo di Cavour; sempre utili le parole di Eugenio Garin che registrava per questo momento un "rovesciamento del fare rispetto al sapere", una "infatuazione pragmatista" che "metteva senza tanti complimenti innanzi la possibilità che ogni fondamento potesse essere distrutto" (Eugenio Garin, Cronache di filosofia italiana, Roma-Bari, Laterza, 1975, vol. I, p. 31). 
nistrazione, stato etico stato di diritto e stato liberale, meridionalismo e anti-meridionalismo, regionalismo e unitarismo, germanesimo e nazionalismo (a cui si intreccia una questione razziale che ha esiti noti, ma che ha radici nel passato). Anche il wagnerismo italiano, molto studiato da altri punti di vista, deve essere inserito in questo ampio quadro; e le reazioni che Verdi ha verso il germanesimo e verso il wagnerismo sono comprensibili solo all'interno di esso.

Si può a questo punto avviare qualche approfondimento, ponendo a confronto due testimonianze della cultura italiana di fine Ottocento: Pasquale Turiello e Verdi stesso. Verdi era stato invitato dal ministro dell'Istruzione Cesare Correnti a presiedere una commissione per la riforma dell'istruzione musicale, ${ }^{11}$ una delle poche occasioni in cui il compositore accettò incarichi ufficiali. Il 22 marzo 1871, due giorni dopo aver terminato i lavori, Verdi scrive all'amico napoletano Cesare De Sanctis: ${ }^{12}$

Se si parlerà meno, e si cercherà di fare di più forse si potrà ottenere qualche buon risultato ... ma anche noi siamo come i nostri vicini [i Francesi che si vantano sempre nous... nous... aveva detto contro la "Grand Boutique" per Don Carlos]! ... Il noi c'invade ... Terribile parola, indizio sempre d'ignoranza e di decadenza ...

La frase che ci interessa è l'ultima, quella che introduce una differenza qualitativa fra l'io e il noi; ma occorre intendere bene cosa significhi qui la punta polemica contro il noi. Verdi ha i piedi piantati nel liberalismo moderato, ${ }^{13}$ nell'etica del dovere sociale di mazziniana

11 Gli atti dei lavori della Commissione furono pubblicati dalla Gazzetta Musicale di Milano, XXVI/22 (28 maggio 1871), "Supplemento straordinario". Una trattazione critica recente di questi eventi si trova in Irene Piazzoni, Spettacolo istituzioni e società nell'Italia postunitaria (1860-1882), Roma, Istituto per la storia del Risorgimento italiano-Archivio Guido Izzi, 2001, cap. II, par. 6 ("Le commissioni Correnti"), pp. 125-133.

12 Carteggi verdiani, a cura di A. Luzio, Roma, Reale Accademia d'Italia e Accademia Nazionale dei Lincei, vol. I, p. 137.

13 Liberalismo moderato non è definizione opinabile e generica, ma indica qualcosa di ben preciso: nel medio ottocento questo sintagma prende a indicare una precisa qualità distintiva del liberalismo rispetto al "liberalismo classico" nato dalla mentalità illuministica e al "liberalismo radicale" (cfr. Appunti della Fondazione Luigi Einaudi, Torino). Da quest'ultimo scaturisce il moderno liberismo, mentre dal liberalismo moderato, con 
memoria, nella "grande idea" del "sacrificio" dell'individuo a favore della collettività: per lui come per tutti i suoi coetanei (De Sanctis, Settembrini, ma anche i cattolici Pellico, Rosmini e Tommaseo) "libertà" è parola che non può andare disgiunta da "dovere sociale". La condanna del "noi" non è allora una professione di individualismo volontarista; semmai è la denuncia della radicale frattura fra io e noi, individuo e società, in favore di un malinteso spirito di gruppo, che in quegli anni nel gergo politico si chiamava "consorteria". E la infrazione dell'ideale di organismo sociale coerente e armonico; è il noi che prelude al settarismo e al nazionalismo aggressivo, opposti all'idea di "umanità" mazziniana. In quegli stessi anni dilaga l'atteggiamento opposto, ugualmente lontano dall'etica di Verdi: il pragmatismo individualista. I best-sellers del momento, Chi si aiuta Dio l'aiuta di Samuel Smiles ${ }^{14} \mathrm{e}$ Volere è potere di Michele Lessona ${ }^{15}$ sono espressioni di questa concezione dell'individualismo. Non è questo il concetto di responsabilità individuale di Verdi, sebbene l'opposta etica del noi sia per lui ancor più pericolosa. Nei suoi melodrammi tra etica e legge sociale è sempre la prima che orienta le scelte dei personaggi positivi, e quando la seconda si impone s'innesca sempre un meccanismo tragico, che obbligatoriamente porta alla catastrofe: così nei Due Foscari, in Trovatore, in Traviata. In Due Foscari come in Aida, per essere più precisi, l'origine del conflitto è la discontinuità fra individuo e collettività, fra bene individuale e interesse comune: nelle parole di Verdi, "il noi ci invade" e toglie lo spazio "naturale" al libero esercizio dell' $i o$.

Se ora affrontiamo il pensiero di Turiello le cose divengono ancor più chiare. Turiello è un discutibile e discusso personaggio che, negli anni fra il secondo Boccanegra e Otello, scrive uno studio che rappresenta molto significativamente, piaccia o no, il clima intellettuale del momento:

ovvi riorientamenti, genererà il liberal-socialismo, ossia la organica equilibratura delle funzioni dello stato con le libertà individuali, qualcosa quindi di molto simile alle ideali aspirazioni di molti personaggi verdiani.

14 Samuel Smiles, Chi si aiuto Dio l'ainta (titolo originale Self-Help with Illustrations of Conduct and Perseverance, 1859), trad. it. di G. Strafforello, Milano, Editori della Biblioteca Utile, 1865 (seguirono altre diciassette edizioni).

15 Michele Lessona, Volere è potere, Firenze, Barbera, 1869; il capitolo IX "Parma”, pp. 287-306, è dedicato interamente a Verdi; il capitolo III "Napoli”, pp. 76124, fu scritto da Pasquale Turiello. 
Governi e governati in Italia. ${ }^{16} \mathrm{La}$ sua posizione contribuisce a dare l'avvio di quella che si chiamò la "psicologia collettiva" o "psicologia delle masse". Ex-garibaldino, passato poi nelle file della Destra, scivolando infine su posizioni sempre più antidemocratiche e centralistiche, Turiello negli anni Ottanta ritiene che il male d'Italia non sia la troppo poca, ma la troppa libertà individuale, frutto di un lungo retaggio tutto italiano a "far da sé", difetto che Turiello chiama "scioltezza". Anche lui ripete la relazione oppositiva noi - io: "nelle risoluzioni e nell'opere sue [dell'Italiano] s'avverte l'io molto più che il noi" ${ }^{17}{ }^{17}$ Non occorre sottolineare come l'accezione ch'egli dà a questi termini è opposta a quella di Verdi; per lui la "scioltezza", l'io individualista, indica l'incapacità, di cui egli accusa l'italiano medio soprattutto meridionale, di sottostare a regole collettive, di sottomettersi a qualunque forma di autorità. Di qui i problemi dello scarso senso di appartenenza, della sostanziale estraneità fra gli individui e il nuovo stato. Contro il liberalismo moderato, egli propone quindi alcuni rimedi a questo problema, soprattutto attraverso l'educazione sin dall'età infantile e adolescenziale (educazione condotta con metodi dedotti dalla vita militare), e conclude: "l'io del giovinetto italiano sia piegato a sentirsi noi" " ${ }^{18}$ Turiello propone poi una teoria storicogenetica di questo carattere "sciolto" degli Italiani: essi sono, dice, frutto di confluenze storiche, non una razza biologicamente unitaria come i germanici; sono una commistione di più razze originarie, differenziate per clima, geografia e storia (ossia percorsi storici e situazioni biologiche di partenza molto differenziate fra settentrione e meridione). Turiello infine accusa i governi di aver commesso sin dal 1860 l'“errore" di troppo rispetto per le libertà individuali, troppo allentamento dei controlli, insomma troppo liberalismo. Inizia qui un lungo filone di critica al liberalismo e al parlamentarismo, che a lungo saranno viste come le due cause principali di decadenza. Ed inizia qui, specularmente, l'ispirazione/ammirazione per il centralismo bismarckiano, per la volontà dello stato, per l'economia "a tappe forzate", per il protezionismo, per l'intervento statale nell'economia, nel sistema bancario e nell'industria.

16 Pasquale Turiello, Governo e governati in Italia, a cura di P. Bevilacqua, Torino, Einaudi, 1980 (ed. or. Bologna, Zanichelli, 1882).

17 Questo atteggiamento viene commentato da Christopher Duggan, La forza del destino. Storia d'Italia dal 1796 a oggi, Roma-Bari, Laterza, 2011, p. 310.

18 Turiello, op. cit., p. 309. 
Evidentemente Verdi non fa parte di questo mondo; egli poggia la sua visione dell'uomo e del mondo sull'individuo, le sue libertà e particolarità all'interno di una società armonicamente coesa (continuità); altrettanto evidentemente Turiello propone una visione che ha in sé inequivocabili segni della teoria dello stato forte, che da Crispi si trasmetterà al Novecento.

Verdi qui rappresenta una mentalità che sta tramontando, davanti a nuovi modelli di stato e società. Il sistema che si afferma fra i Settanta e gli Ottanta è formato da una costellazione di componenti, per la maggioranza assimilate dal modello germanico, e ovviamente guardate con aperta diffidenza da Verdi. È il modello tedesco in politica come in economia, la realpolitik contro l'idealismo liberale classico, un nuovo statalismo chiaramente dedotto dal modello del Machtstaat bismarckiano, con forti ricadute anche nei campi dell'economia e della cultura.

Un classico studio di storia politica, ${ }^{19}$ trattando di questo argomento, parla di "individualismo cavouriano" e del suo "liberalismo pragmatico, che lo fa in fondo poco sensibile alla esaltazione dello stato"; in queste parole è riassunta la distanza che separerà i due orientamenti: quello di Verdi che, come Cavour, pone limiti naturali e invalicabili al noi come forma-stato, e quello di Turiello e Lombroso (ma anche di Crispi e dei socialisti Costa e Turati), che invece per opposte vie antepongono la struttura istituzionale alle libertà inalienabili dell'individuo.

Le idee di Turiello ebbero vasta risonanza; già due anni dopo il filosofo della storia Nicola Marselli sosteneva che i difetti di "scioltezza" caratterizzavano tutti gli italiani, non solo meridionali, e che perciò ancor più occorresse uno stato forte. ${ }^{20}$ Più tardi anche Giuseppe Salvioli condivide l'accusa all'individualismo e introduce la polemica antiborghese, che aumenterà rapidamente: ${ }^{21}$

tutti i codici di questo secolo sono fatti per una società borghese, da giureconsulti per origine ed educazione borghesi, cioè rimpinzati di diritto romano, e che dell'economia sociale non conobbero che quanto riferivasi all'accumulamento e alla conservazione della ricchezza.

19 Alfredo Capone, Destra e sinistra da Cavour a Crispi, Milano, Tea, 1981, p. 113.

20 Nicola Marselli, Gl'Italiani del Mezzogiorno, Roma, Sommaruga, 1884.

21 Giuseppe Salvioli, I difetti sociali del codice civile in relazione alle classi non abbienti e operaie, Palermo, Tipografia dello Statuto, 1891. 
insomma un sostenitore dello "sviluppo economico a tappe forzate", basato sulla forza dello stato e sulla potenza dell'imprenditorialità individuale, sul modello germanico-bismarckiano, precisa espressione di quella deutsche Bewegung di cui il wagnerismo è un altro aspetto.

Un altro criminologo positivista, Enrico Ferri, militante dell'Estrema e poi fra i socialisti, aggiunge un chiarimento alla questione del moderno individualismo aggressivo e disgregante il tessuto sociale, comune secondo lui a tutte le nazioni europee, ma particolarmente pernicioso in Italia, dove la morale collettiva non è ancora formata. Ecco come Ferri sintetizza il problema: ${ }^{22}$

[il] secolo XIX si chiude col predominio riconosciuto dalla scienza positiva alla collettività umana contro l'apoteosi dell'individuo che chiudeva il secolo XVIII [e apriva il successivo]; ed alla quale invano tentano ora ritornare alcuni artisti, dalle pose più o meno anarchico-individuali. Ma è facile a chi sente di avere una personalità spiccata, il persuadersi che solo gli "uomini eletti", precursori dei "super-uomini" avvenire, abbiano un qualche valore nella infinita ed anonima turba dell'umanità - e l'egotismo non è che una degenerazione del senso della personalità umana. Ma oltre i vaneggiamenti orgogliosi ma miopi dell'egotismo, la conclusione vera e completa è che come la collettività si evolve sotto l'impulso del pensiero e dell'azione individuale, così l'individuo rimane impotente e non è neppur concepibile nella realtà della vita senza la base umana della collettività, a cui esso deve, malgrado le sue illusioni più o meno patologiche, tutto ciò che costituisce il fondamento del suo pensiero e della sua vita.

Ferri sente il problema, come lo sentiva Verdi, e come quest'ultimo sebbene da opposta prospettiva avanza una critica sia all'individualismo sia allo statalismo astratto, da socialista critico qual era.

Molti di coloro che ho fin qui nominato partono in gioventù come entusiasti seguaci di Garibaldi, il principale simbolo del sacrificio dell'individuo a favore dell'interesse collettivo; tutti, poi, vanno verso un socialismo umanitario; ma alla fine alcuni come Turiello prendono la direzione dell'autoritarismo, dello stato forte, dell'anti-democratismo, altri invece convergono verso il neonato partito socialista, non sempre in modo rettilineo. È lo spirito che da Smiles e Lessona arriva a Giuseppe Sergi, Scipio Sighele e i Nazionalisti di Corradini.

22 Enrico Ferri, I delinquenti nell'arte, Genova, Libreria Editrice Ligure, 1896, p. 113. 
L'accusa "politica" all'individualismo, alla "scioltezza", rimane come luogo ricorrente fino al nuovo secolo, a destra come a sinistra; tanto che nel 1911 Benedetto Croce scrive:

la decadenza del sentimento dell'unità sociale $[\ldots]$ va alla pari [con] una generale decadenza del sentimento di disciplina sociale: gli individui non si sentono più legati a un gran tutto. [...] La buona individualità [...] ha ceduto il posto alla cattiva individualità. ${ }^{23}$

Croce è in questi anni più vicino all'idea di stato etico, che a quella di stato liberale; eppure la sua allusione alla "buona individualità" non è lontana dall'idea di Verdi e ricorda quella borghesia responsabile e attiva, sia pur basata sul valore dell'individualismo, che anche Leone Carpi aveva definito "vero nerbo della nazione". ${ }^{4}$ D'altronde, terminata l'onda positivista, ossia la "infatuazione pragmatista" di cui parlava Garin, con la ripresa dei neo-idealismi novecenteschi anche il rapporto individuo-collettività incontra nuove evoluzioni.

\section{DEUTSCHE BEWEGUNG}

Indissolubilmente legata a questa, come già detto, è la questione del germanesimo, la nuova corrente di opinione pubblica, economia, politica e cultura, che va sotto i nomi di "via prussiana" (Procacci) o deutsche Bewegung. La diffidenza verso questo movimento da parte della generazione di Verdi inizia assai precocemente; Carlo Tenca per esempio scrive a Clara Maffei sin dal 1870: ${ }^{25}$

23 Benedetto Croce, Fede e programmi [per la morte di A. Fogazzaro], in "Giornale d'Italia" 12 settembre 1911, si cita dall'antologia 1911. Calendario italiano, a cura di Lorenzo Benadusi e Simona Colarizi, Roma-Bari, Laterza, 2011, p. 206-211: 209.

24 Leone Carpi, L'Italia vivente. Studi sociali, Milano, Vallardi, 1878, p. 503. Per questi argomenti cfr. Alberto Mario Banti, Storia della Borghesia italiana. L'età liberale, Roma, Donzelli, 1996, pp. 227-229.

25 Lettera di Carlo Tenca a Clara Maffei, Andorno 7 settembre 1870 (Carteggio Tenca-Maffei. Tomo Primo: 1861-1871, a cura di L. Jannuzzi, Milano, Ceschina, 1973, p. 245). 
la Francia [...] è soggiogata da una intelligenza preponderante, dalla forza che scaturisce da un ordine di istituzioni e di idee diverse e più efficaci. È una nazione che si sovrappone ad un'altra e le toglie di mano l'indirizzo della civiltà. La supremazia latina se n'è andata, ed ora il mondo è della stirpe germanica.

In questa lettera Tenca istituisce un'opposizione fra la "missione educatrice in Europa" che la Germania si è implicitamente attribuita, e la "civiltà" e "libertà" di Parigi, ${ }^{26}$ Tenca intende precisamente il principio del liberalismo classico, che a lui come a Verdi proviene dalla tradizione risorgimentale: "libertà nel cuore" ossia individuale, "libertà nelle istituzioni" ossia ordine statuale basato sulla salvaguardia delle libertà inalienabili dell'individuo, non stato superiore agli individui e quindi limitante le loro libertà. È precisamente questo doppio livello organico della libertà di diritto che Tenca, come Verdi, ha assimilato dalla sua fase idealistica e che gli rimane come rimpianto mai realizzato in Italia.

L'influenza tedesca non ha in sé nulla di negativo, non quel senso di disfatta epocale che trapela da alcune note lettere di Verdi ${ }^{27} \mathrm{ma}$ ad essa diversi pensatori connettono aspetti demologici, etnotipici e antropoticipi che troppo facilmente possono degenerare in questioni razziali. Secondo Turiello, Lombroso, Sergi, Ferrero, Pareto, Sighele ed altri, poi, l'individualismo degenere, dannoso perché anti-sociale, è tipico del carattere $^{28}$ dei popoli mediterranei; i popoli continentali, gli Arii come sono allora definiti, sarebbero invece più collettivisti, per cui sarebbero maggiormente adatti alla costituzione del moderno stato forte. Giuseppe Sergi è forse il più chiaro su questo argomento, quando afferma che la "psiche etnica" degli italiani è nata da misto di due razze: Arii

26 Ivi, p. 247.

27 "Brutta cosa per la nostra natura aver a che fare con quella gente di ferro e senza cuore. Lo strano si è che noi non solo diciamo 'aiutateci nel caso col vostro potente braccio', ma li ammiriamo, li aduliamo, li imitiamo anche dove non sono da ammirarsi né da imitarsi. Lasciamo da parte le cose teatrali di cui non voglio né devo parlarne, [...] ma letteratura, scienza, medicina etc. etc. tutto è tedesco" (lettera di Verdi a Opprandino Arrivabene, 25 ottobre 1873, in Verdi intimo. Carteggio di Giuseppe Verdi con il conte Opprandino Arrivabene (1861-1886), a cura di A. Alberti, Milano, Mondadori, 1931, p.162). "Noi non abbiamo più né letteratura, né arti, né scienze nostre. Tutto è forestiero, e siamo già a due terzi non più italiani nemmeno politicamente" (lettera di Verdi a Opprandino Arrivabene, Genova, 8 dicembre 1881, ivi, p. 291).

28 Emerge anche in questi anni la distinzione fra "carattere" e "identità": il primo naturale, la seconda volontaristica. 
o "razze celtiche" di origine euro-asiatica, Italici o Mediterranei di origine euro-africana. ${ }^{29} \mathrm{La}$ differenza fra le due razze è proprio nella collettivizzazione "naturale" e non forzata della prima (unità sociale: "l'individuo si fonde nell'aggregato senza alcun sacrifizio"), contro l'indole meridionale dove "ogni individuo vuole emergere dalla massa sociale".

Guglielmo Ferrero, seguace di Lombroso (di cui sposò la figlia Gina), è sulla stessa linea di Sergi; anche lui ricorre alla distinzione razziale: le "razze germaniche" (intende Inglesi, Tedeschi e NordAmericani) sono secondo lui dotate di un senso dello stato più forte, un più alto senso del dovere, una predisposizione al lavoro organizzato e meccanizzato, attributi necessari nelle società moderne. ${ }^{30}$ Di contro, le razze meridionali-latine sono sensuali e ingegnose ma poco energiche: $i$ loro difetti caratteriali si riassumono in anarchia morale e cesarismo, ossia in una mancanza di responsabilità individuale davanti al corpo sociale (Ferrero non dice questo in modo esplicito, ma lo si può dedurre leggendolo dalla prospettiva del liberalismo di Verdi).

E proprio su questo pregiudizio che, allo scopo di saldare la giovane nazione già indebolita da autonomismi e personalismi, con i governi delle sinistre (Depretis, Cairoli, Crispi) il modello tedesco diventa fenomeno pervasivo e trasversale. In questa situazione possono essere comprese la profondità e la longevità del wagnerismo italiano, che sarebbe del tutto fuorviante estrarre dal fenomeno sociale della deutsche Bewegung per ridurlo a fenomeno teatrale. Né si potrebbe capire la radicale estraneità del liberale moderato Verdi, se la riducessimo a una polemica artistica, che certo non era nelle sue intenzioni.

Al centro di questo processo si colloca la svolta di politica internazionale guidata da Crispi e dai suoi governi, svolta che chiude un secolare rapporto economico, politico, culturale, artistico con la Francia, per girare l'ago della bussola verso Berlino. La svolta inizia negli anni Settanta e giunge a maturazione nel 1887 (l'anno di Otello), quando Crispi diventa capo del governo e rinnova la Triplice Alleanza, ora dive-

29 Giuseppe Sergi, Per l'educazione del carattere. Pagine di psicologia sociale e consigli direttivi, Torino, Camilla e Bertolino, 1885, p. 100, cit. in Patriarca, Italianità cit., pp. 92-93; la scelta terminologica rimanda a Gioberti, ma naturalmente senza alcuno dei suoi fondamenti cattolico-neoguelfi.

30 Guglielmo Ferrero, L'Europa giovane. Studi e viaggi nei paesi del Nord, Milano, Treves, 1897, pp. 420-421. 
nuta più favorevole all'Italia rispetto al primo quinquennio. È davvero la fine di un'epoca. Ovvio, non intendo dire che il tardo Verdi sia influenzato dal germanesimo né dal wagnerismo, tesi d'altronde assai vecchia e liquidata da tempo. Intendo invece che tanto la produzione del tardo Verdi (dal Requiem ai Pezzi sacri) quanto il wagnerismo sono espressioni di quel movimento collettivo che accompagna il difficile processo dell'Italia borghese dalla Sinistra storica all'età giolittiana, con tutte le contraddizioni che lo distinguono: industrializzazione a discapito del meridione, inurbamento a discapito delle zone rurali, statalismo a discapito del liberalismo, imperialismo a discapito dei movimenti umanitari, positivismo e cattolicesimo, germanesimo e nazionalismo.

L'espressione deutsche Bewegung indica un movimento di idee e di prassi (comportamentale, sociale, politica) fatto di molte diverse componenti, fra cui lo statalismo, la prevalenza dell'amministrazione sul legislativo, il protezionismo economico, il volontarismo, il militarismo e l'esaltazione della forza fisica, esercitata anche nella vita quotidiana (addirittura l'insospettabile De Sanctis come ministro dell'istruzione arriva a promuovere nella scuola dell'obbligo più educazione fisica che letteratura). Ritrae questa situazione una lettera del 12 marzo 1877 in cui Piroli scrive a Verdi della "nuova scuola" politica di Depretis, Nicotera, Crispi e Cairoli: ${ }^{31}$

La nuova scuola, ${ }^{32}$ o come già la chiamano la nuova consorteria progressista si compone in gran parte di gente ignorante che presume progredire senza conoscere il punto di partenza, e d'altre parte la necessità in cui è il Ministero di mantenere una parte delle promesse che non potrà effettuare se non in una misura molto limitata, voi vedete che la prospettiva si fa sempre

31 Giuseppe Piroli a Verdi, 12 marzo 1877, in Carteggi vol. IV, p. 124 (Piroli era deputato a Roma e teneva informato Verdi delle novità maggiori, senza sottrarsi a scambi di opinione politica con il maestro).

32 Alfredo Capone, Destra e sinistra cit., p. 253; si tratta di una nuova scuola di economia industrialista che, seguendo il modello germanico di Adolf Wagner e di Lujo Brentano, teorizza l'avversione al liberismo cavouriano e moderato tipico del sistema italiano, contro lo sviluppo agrario, per il processo tecnologico a favore dell'industria e l'azione dello stato forte. A pieno favore dell'industrializzazione con capitale statale, l'esordio della "scuola" avviene nel 1870 quando Luzzatti ipotizza al Congresso della Società italiana di Economia politica il sostegno di stato e banche all'industria. Questa corrente "statalista" prende poi forza soprattutto a partire dal 1874, quando Luzzatti, con Rossi, Robecchi e Scialoja, conclude la sua inchiesta sulle condizione dell'industria in Italia. 
più scura ed incerta. [...] Relativamente alla vostra gita a Cologne vi dirò che circa un mese fa il Sella mi aveva fatto pregare di scrivervi per sapere se andavate e ad interpormi per rappresentarvi tutti i motivi che anche politicamente facevano desiderare il vostro intervento a quella solennità musicale.

Dalla frase finale capiamo poi che Verdi era effettivamente (e involontariamente) dentro questo disegno di avvicinamento alla Prussia, e che Quintino Sella si rendeva conto del valore diplomatico della partecipazione di Verdi al $45^{\circ}$ Niederrheinische Musikfest organizzato da Ferdinand Hiller a Colonia, dove Verdi diresse il suo Requiem il 21 maggio 1877. Wagner, anche senza conoscerlo sufficientemente, ${ }^{33}$ diviene tratto culturale di questo movimento; e Verdi ne è preoccupato non certo per sé, per timore di rimanere attardato o fuori gioco; e non certo per le esecuzioni wagneriane nei teatri italiani; la sua preoccupazione è ben più radicale, più generale, più per gli altri che per sé:

Oggidì non si trovano più né Maestri né alunni che non siano attaccati di Germanesimo, e neppure si potrebbe formare una commissione esente da questa malattia. ${ }^{34}$

Quest'invasione d'un'arte forestiera ha acciecato noi tutti in modo che c'impedisce di vedere come i Tedeschi, facendo della musica tedesca, sono nel vero ed hanno ragione. Noi invece imitandoli abbiamo negato l'indole nostra, facendo musica senza carattere italiano, ibrida e bastarda. ${ }^{35}$

La stessa concezione del liberalismo classico, che nel campo sociale vuole continuità fra bene comune e interesse individuale, si applica così alla composizione melodrammatica, dove lo stile individuale dovrebbe secondo Verdi trovare espressione in continuità con il

33 In Italia arrivano dapprima solo le opere romantiche; la Tetralogia arriva nel 1883 a Bologna, Torino e Roma grazie alla compagnia di Angelo Naumann, Tristan arriverà solo nel 1888 a Bologna direttore Giuseppe Martucci, i Meistersinger nel 1889 alla Scala sotto la guida di Franco Faccio.

34 Lettera di Verdi a Piroli, Genova, 2 febbraio 1883, Copialettere pp. 319-320. Nella minuta datata 21 gennaio la forma era lievemente diversa, uguale la sostanza: "Il germanesimo di invade; i maestri stessi seguono la corrente; non credono più all'arte italiana e non sanno più scrivere italianamente. Così il nostro teatro muore" (Ibid.).

35 Lettera di Verdi al ministro dell'Istruzione Guido Baccelli, Genova 4 febbraio 1883, Copialettere p. 321. 
"carattere" di una nazione (fatto di tradizione e convenzioni consolidate). L'incosciente accettazione della deutsche Bewegung rischierebbe invece, nella sua opinione, di interrompere questa continuità. E in questa Bewegung sono coinvolti tutti; ogni presa di posizione pubblica, ogni scelta sia politica che artistica è esposta al rischio di essere strumentalizzata. Per cui Verdi non tollera che i collaboratori di vecchia data, coloro che hanno attraversato i decenni della lotta idealistica per la libertà e la autodeterminazione nazionale, si lascino prendere nel giro di queste strumentalizzazioni; peggio poi se lo fanno inconsapevolmente, come accade al povero "ragazzo" Angelo Mariani, con i due episodi della Messa per Rossini (rivendicazione di una storia nazionale) e delle prime wagneriane a Bologna e Firenze (1871-72). ${ }^{36}$ È per questo motivo che anche l'allusione all'utilità politica della sua trasferta a Colonia, caldeggiata da Sella, viene presa da Verdi con una certa suscettibilità: "Andrò a Cologne, e partirò d'Italia verso il 10 maggio. Non capisco quello che vi disse Sella. Questi Festivals in Germania hanno una grande importanza artistica ed io non cerco di più".

\section{DELUSIONE E DISILLUSIONE}

Individualismo (positivo o negativo) e Via prussiana; la terza componente di questo scorcio di secolo, tanto evidente sia nel contesto nazionale sia nella personale vicenda intellettuale di Verdi, è quella del pessimismo generalizzato, della demoralizzazione, della perdita di fiducia nel futuro, legata alla delusione seguita agli eroici sforzi compiuti nei decenni passati. Ė lo stesso Verdi a definirsi "il più gran pessimista che esista" ${ }^{38}$ Con una significativa contraddizione, questo atteggiamento capovolge la fiducia nel progresso positivistica; per cui, come nel romanzo di Pirandello I vecchi e i giovani, si trovano si fronte i disillusi e i cinici. Stefano Jacini, già ministro di Cavour, scriveva a Émile de

36 Per i risvolti politici di queste vicende rimando a Carlo Matteo Mossa, Una "messa" per la storia, in Messa per Rossini. La storia, il testo, la musica, in "Quaderni dell'Istituto di Studi Verdiani” 5 (1988), pp. 11-78; e al mio Verdi e Mariani, in Giuseppe Verdi, genovese, a cura di R. Iovino e S. Verdino, Lucca, LIM, 2000, pp. 33-60.

37 Lettera di Verdi a Piroli, 27 marzo 1877, Carteggi vol. IV, p. 125.

38 Ibid. 
Laveleye (economista francese di tendenza socialista, che aveva pubblicato nel 1880 Lettres d'Italie; i suoi resoconti erano relativi al 1878): "Vi è [nell'Italia attuale] qualche indizio che si sia destinati a languire di tabe senile". ${ }^{39}$ Commentando questo passo, Turiello concludeva:

si può rilevare da alcuni anni un senso crescente di sconforto, comune a tutti $\mathrm{i}$ partiti, verso le nostre condizioni presenti. [...] citeremo in questo senso le opere del Minghetti, del Sonnino, del Franchetti, del Torraca, del Santamaria, del Zini, del Lombroso, del Dotto, del Jacini, dell'Argentino, del Cantalupo". ${ }^{40}$

La posizione di Verdi è in linea con i tempi, o almeno con una larga parte di italiani che vivevano quel momento post-risorgimentale: Verdi appartiene ad una generazione con la quale condivide la delusione e la preoccupazione per l'aumento del potere politico della borghesia, o meglio di un tipo di borghesia che non aveva assimilato alcun fondamento del liberalismo moderato precedente. Il pessimismo di Verdi, poi, si nutre anche di un timore radicato e diffuso nei suoi coetanei verso i nuovi movimenti di massa, quello socialista in particolare. E con questi movimenti sociali va modificandosi la considerazione e l'incidenza politica dell'entità "popolo", che poco alla volta sta conquistando non solo diffusione sociale, ma anche rappresentanza politica: dal "popolo" mazziniano depositario di tutte le migliori qualità umanitarie, si passava ora al "popolo" minaccioso degli scioperanti e delle proteste di piazza; il paternalismo era sostituito dal timore dello scontro di classe che si faceva via via più violento.

Che poi le tarde opere verdiane possano oggi essere lette anche estraendole e completamente isolandole da quel contesto di psicologia collettiva pessimistica, è questione che si pone su un piano diverso. Che il Boccanegra o il Falstaff nascano da un progressivo ritiro nell'isolamento e nella disillusione non è altro che il segno del momento, che Verdi, sempre attento al contesto a cui si rivolge, condivide con molti suoi coetanei. Il progressivo indebolimento del liberalismo moderato, insieme all'avanzare della mentalità positivistica, che momentaneamente limita l'idealismo precedente, insieme all'ascesa

39 Turiello, Governi e governati cit., p. 257.

40 Ibid. (corsivo mio). 
delle due grandi forze popolari di massa (la democrazia cristiana e il socialismo), incidono profondamente sulla psicologia di quella generazione. Se nasce un capolavoro come Otello è anche in conseguenza di questo movimento dell'opinione pubblica e del contesto sociale, indipendentemente dalle intenzioni di Verdi. Letto in questo modo Otello come Aida o lo Stabat mater hanno valore di documenti della mentalità diffusa in quel momento, senza che ciò nulla tolga al valore artistico. Certo è, tuttavia, che questa funzione di testimone di una mentalità non può essere riscontrata sui documenti, mai troveremo una lettera in cui Verdi dice: "in Otello ho voluto rispecchiare la delusione per i governi di Depretis e Crispi" ; ${ }^{41}$ ma ciò non deve rendere cieca l'attività dello storico, precludendo la comprensione di legami che a me sembrano indiscutibili. Se Otello è un capolavoro, lo è anche perché in esso risuona il rimpianto di quell'idealismo che dava il tono alle opere precedenti. È un rimpianto per qualcosa che non può più esserci, in cui Verdi non crede più: Desdemona e Otello muoiono come tanti altri eroi ed eroine di Verdi, ma contrariamente a questi ultimi, Desdemona e Otello muoiono senza alcuna ragione, non per un'idea, non per un sacrificio a favore di altri, non per una fedeltà a un principio morale, non per denunciare un sopruso o un'ingiustizia: "la Morte è il Nulla", appunto. È la melanconia del nulla; esattamente quella "tabe senile", quel "crescente senso di sconforto", ma con una profondità emozionale che ci fa vivere dall'interno la mentalità di quel momento, per meglio percepire cosa accadeva nell'opinione pubblica in quei turbolenti decenni di fine secolo. Nessun documento potrebbe fare altrettanto con uguale evidenza.

\section{VERDI E WAGNER}

Se ora rileggiamo alcuni dei giudizi su Verdi e Wagner, che compaiono a fine secolo nella stampa non musicale italiana ritroviamo i

41 E tuttavia non mancano sue lettere contro la nuova classe dirigente: "Poveri noi, in che mani siamo! ... O ambiziosi, o ignoranti. A me poco importa dei bianchi [ministeriali], dei rossi [l'Estrema di Bertani, i radicali di Cavallotti], dei destri, dei sinistri, ma vorrei degli uomini capaci e pratici" (Verdi a Piroli, 22 giugno 1879, Carteggi vol. IV, p. 140). 
medesimi argomenti. Certo, alcuni giornalisti specializzati come Filippo Filippi tentano dagli anni di Aida di mettere a fuoco alcune delle novità stilistiche dell'ultima produzione di Verdi (Filippi è il primo a vedere l'equilibrata coesistenza di vecchio e di nuovo nel linguaggio musicale dell'opera) e alludono a labili tracce wagneriane. Più radicalmente Eduard Hanslick, dopo la prima esecuzione dell'opera a Vienna nel 1875 , indica una originale indipendenza da influenze wagneriane. ${ }^{42} \mathrm{Nel}$ panorama italiano, poi, difficilmente vengono discussi i grandi concetti wagneriani, come melodia infinita, motivi conduttori, opera d'arte totale, né si avverte uno sforzo di comprendere la novità della drammaturgia, per cui eventuali accuse di wagnerismo a Verdi rimangono sempre un po' esteriori.

Qui tuttavia, piuttosto che le riflessioni su questioni tecniche musicali o di drammaturgia, mi interessa vedere alcune testimonianze su quale valore sociale, quale collocazione nell'opinione pubblica avessero assunto i due artisti. Ad ognuno di essi, com'è a questo punto prevedibile, viene assegnato un ruolo, una funzione che travalica i limiti dell'arte. Individualismo, genialità unica e indipendente, valore di voce collettiva, modello liberale moderato, modello indipendente self-help: com'è chiaro tutto ciò torna nella valutazione (e nella strumentalizzazione) delle due figure, assegnando loro un valore testimoniale extraartistico, sicché divengono fattori di costruzione di identità sociali generali. È anche troppo nota la lettura del Verdi come voce del popolo italiano, colui che

[...] trasse i suoi cori

dall'imo gorgo dell'ansante folla.

Diede una voce alle speranze e ai lutti.

Pianse ed amò per tutti.

(d'Annunzio)

42 "In questa nuova creazione risplende l'appassionata eloquenza e la forza drammatica, che caratterizzavano già le precedenti opere di Verdi, artisticamente controllata e purificata dalle scorie, in un'arte di catarsi estetica. Nondimeno si tratta del vero e completo Verdi. Un'imitazione di Richard Wagner, come s'è sostenuto in molte critiche, qui non si trova. [...] In Aida non c'è una sola battuta per cui l'italiano sembra direttamente indebitato con il tedesco" (Eduard Hanslick, Die moderne Oper. Kritiken und Studien, Berlin, Allgemeiner Verein für Deutsche Literatur, 1885, pp. 252 4, traduzione mia). 
Quest'enfasi, calata nel quadro della discussione su individualismo e collettivismo, stato forte e stato liberale, non può affatto intendersi solo come un volo poetico, ma rappresenta una precisa interpretazione funzionale a una ideologia. L'aspetto poetizzante e nostalgico dei versi dannunziani non riesce a nascondere sotto la maschera populista-nazionalista un fondamento centralista e persino totalitario. Il tardo Verdi infatti non "pianse ed amò" affatto "per tutti", ma per denunciare, dall'opposta posizione di isolamento e ritiro solitario, la direzione pericolosa verso cui molti stavano incamminandosi.

Un giudizio particolarmente significativo, dal mio punto di vista, è quello di Cesare Lombroso, interessante qui anche per lo spazio che a lui abbiamo assegnato nelle precedenti pagine; e ancor più interessante perché è lui stesso a proporre un raffronto Verdi-Wagner applicando ad entrambi il medesimo parametro. Enrico Ferri, nell'emettere un giudizio sul genio artistico dei "degenerati” Baudelaire, Zola, Ibsen, Dostoevskij e Wagner, afferma di non prendere in considerazione l'opera d'arte, ma l'autore e le sue personali degenerazioni. ${ }^{43}$ Questa prospettiva è la stessa per cui Lombroso non studia il crimine, non ipotizza una giustizia criminale uguale per tutti, ma studia i criminali perché ne venga giudicato non solo l'atto ma soprattutto il contesto d'azione che l'ha prodotto. Perciò i seguenti giudizi lombrosiani non sono sulle opere, come la giustizia positiva non giudica i crimini in astratto; ma sono giudicati e posti in raffronto gli autori, esattamente come la giustizia positiva giudica i criminali, il loro contesto e la loro costruzione esistenziale. Questa non sembri una ovvietà, perché già Lombroso era consapevole di quanto le sue riflessioni fossero sottoposte a distorsioni e critiche strumentali quando scriveva: ${ }^{44}$

è proprio allo stesso modo come i giuristi delle vecchie scuole giuridiche si scandalizzavano delle applicazioni antropologiche-criminali nei casi in cui il delitto era più orrendo, più bestiale, e si rifiutavano ad ammettere che allora appunto più mostrasse doverne esser anomalo l'autore, e più giustificarne l'indagine antropologica; e così io sento ripetermi: $\mathrm{Oh}$ ! con che coraggio volete trovare anomalo un genio che vi ha disegnato le logge del Vaticano, o vi ha scolpito il Mosè, o vi ha rivelato il nuovo mondo? E non capiscono che non è l'indagine critica dell'opera che ci preoccupa, sì quella del loro autore, in rappor-

43 Enrico Ferri, I delinquenti nell'arte cit., p. 106-108, dove cita e commenta uno studio di Max Nordau su Wagner "degenerato".

44 Cesare Lombroso, L'uomo di genio cit., p. 7. 
to con essa: che anzi, quanto più quella è sovrumana, più è probabile sia anomalo questo.

Un principio questo che ricorre negli scritti di molti suoi allievi e collaboratori, dal citato Enrico Ferri a Mario Carrara e Luigi Roncoroni (citati nella prefazione a L'uomo di genio). Sottolineata quindi la avalutatività delle scienze positive applicate all'arte, Lombroso detta, sollecitato dalla Gazzetta musicale di Milano dopo il successo del Falstaff, una sua lettura della figura di Verdi (e sia chiaro, egli parla della figura, dell'esemplare inteso come oggetto di studio comparativo nei confronti della generalità statistica, non del Falstaff come opera d'arte): ${ }^{45}$

La forma dei lavori scritti in vecchiaia da Carlyle, Dickens, Giorgio Elliot e Tennyson potrà essere eccellente, ma è spesso una ripetizione, sotto nuova veste, di ciò che essi avevano già detto da giovani più volte. E l'opere geniali di Burns, d'Alfieri, di Darwin se furon dettate nell'età matura, furon preparate o concepite nella giovinezza: ed è l'epoca del concepimento quello che più importa.

Queste ragioni, questi pretesti con cui si viene a spiegare o piuttosto direi quasi a inforsare la tardività del genio, io lo confesso, mancano del tutto al Verdi, nel quale del resto mancano tutti gli altri caratteri della nevrosi del genio; non resterebbe altra spiegazione se non quella dell'anomalia che è propria del genio, e che ordinariamente si associa alla precocità, qui invece si esplica colla tardività come in alcuni anomali che presentano le suture ancora aperte del cranio dopo i 70 anni, oppure che presentano una attività sessuale pari o anche maggiore di quella dei giovani.

Però è più onesto il confessare che le spiegazioni non si trovano, che l'anomalia è così grande, così straordinaria da imbrogliare chi di queste ricerche fece uno studio speciale.

Quando penso che il Falstaff è una concezione tutta festosa e allegra, e che nella vecchiaia si han piuttosto le note lugubri, e che in nessun modo le si può dire l'ultima elaborazione di un lavoro pensato in gioventù, come sono di solito le opere dei vecchi, confesso che mi sfugge ogni spiegazione possibile.

Augusto Guido Bianchi, collaboratore di Lombroso, ${ }^{46}$ ne riprende le posizioni in una polemica a distanza con Max Nordau su Wagner,

45 Cesare Lombroso, Il fenomeno psicologico di Verdi, in "Gazzetta musicale di Milano", LVIII/10 (5 marzo 1893), pp. 159-161; l'articolo è datato "Torino, 15 febbraio 1893 ", una settimana dopo la prima alla Scala (corsivi miei).

46 Dalla loro collaborazione uscirono diversi studi, uno fra i quali dedicato alla 
pubblicata ancora sulla Gazzetta di Ricordi: "al grande successo incontrato dal Wagner non è certo indifferente lo stato morboso di quest'epoca nostra" ${ }^{47} \mathrm{Il}$ wagnerismo italiano, perciò, sarebbe funzione di uno stato di patologia collettiva, di psicosi morbosa, per cui l'esaltazione ripetitiva dei suoi drammi musicali incontrerebbe una particolare predisposizione dell'uditorio. Come si vede, anche qui non si tratta di valutare l'opera di Wagner, ma di collocarla come testimone-interprete di un psicologia dominante:

Il genio per Cesare Lombroso rappresenta sempre una anormalità: è un'esagerazione di una facoltà intellettuale, la quale genera una sproporzione, L'opera del genio è qualcosa d'impulsivo [...] io credo che nella sostanza un fatto permanga evidente, risollevando una domanda; è il genio preferibile all'ingegno e non rappresenta questo un'attività progressiva molto più sana e biologica? [...] Il Falstaff è una prova, giacché mentre molti giovani continuano a smaniarsi in una servile imitazione, e in rigide applicazioni di principi del Wagner, affatto personali, il gran vegliardo, Giuseppe Verdi, nel maraviglioso equilibrio delle sue qualità superiori ha già operato, naturalmente, la capitalizzazione di quelli fra i criteri del Wagner che erano accettabili per il progresso della musica teatrale. [...] Questo mi piace di constatare $[\ldots]$, come modesto ma coscientemente entusiastico omaggio di studioso, a questa sana personificazione della genialità italiana. ${ }^{48}$

Bianchi qui probabilmente interpreta il pensiero dello stesso Lombroso, il quale infatti pochi anni dopo affronta il problema Wagner, dandone una lettura patologica, in linea con la sua teoria del genio come deviazione e degenerazione. Oltre a Bianchi, qui Lombroso si ricollega ad alcune affermazioni sulla genialità patologica di Wagner proposte da Luigi Roncoroni. ${ }^{49} \mathrm{E}$ su Wagner lo stesso Lombroso proietta tutte le devianze riscontrate statisticamente nelle sue ricerche, quasi

creatività del genio: La patologia del genio e gli scienziati italiani. Inchiesta a proposito del caso di Guy de Maupassant, Milano, Kantorowicz, 1892.

47 A.G. Bianchi, Wagner e la psichiatria, in "Gazzetta musicale di Milano" XLVIII/10 (5 marzo 1893), pp. 163-166 (II parte).

48 Ibid.

49 Luigi Roncoroni, La lotta per la vita e per l'arte, in "Rivista moderna di coltura" I/3-5-6, (1898); anche Roncoroni era nell'area di influenza di Lombroso, di cui dal 1894 era stato assistente, quando questi era direttore del manicomio di Torino. 
a farne un paradigma della sua teoria; operazione, ovviamente, neppure stavolta estranea a intenzioni politiche più o meno esplicite:

Riccardo Wagner ci dimostra la grande sua precocità nell'ingegno e la violenza delle emozioni nella megalomania, nel "gigantismo monoemozionale" che lo caratterizzarono poi sempre. Fin dall'età che per gli altri ragazzi è destinata ai giuochi dell'infanzia, si rivelarono in Wagner la passione per l'arte e per la gloria, e il tratto caratteristico della impossibilità di battere la via comune, di assoggettarsi alle leggi stabilite. Spirito fantastico, dominato da un misticismo esagerato, "faceva dei sogni in pieno giorno" [...]. Anche il suo egoismo è dimostrato dalla sua relazione con Meyerbeer, e con molti amici. La violenza delle sue emozioni è rivelata dall'importanza che attribuiva alle critiche altrui: Mi si lodi, o mi si biasimi - scriveva - è come mi si pugnalassero le intestina. Agilissimo, saliva sugli alberi più alti del giardino, ed era vanitosissimo della sua agilità. Nei momenti di eccitamento sembrava in preda alla febbre; tutto pieno di fuoco, incapace di star fermo, saltava, si dimenava, agitava a destra ed a sinistra le sue braccia di ragno; le parole uscivano dalla sua bocca a fiotti, disordinate [...]. Vero zoofilomaniaco ebbe amicissimi 13 cani, a molti dei quali elevò tombe. [...] Soffrì spesso di cefalea: "I miei nervi", scrive "sono sempre eccitati e stanchi, mai in riposo: il mio male è incurabile". [...] Uno dei suoi tratti caratteristici fu l'instabilità delle idee e della condotta, rivelata specialmente dalle sue opinioni politiche e dai suoi atti, come pure dai viaggi frequentissimi spesso fatti senza alcuna necessità. [...] Ebbe, secondo il Nisbet, accessi epilettici prima di morire. Il rapporto tra l'ispirazione generale e l'accesso epilettico appare alle parole stesse del Wagner sul suo estro: "I miei occhi si oscurano, il mondo mortale scompare, e l'ispirazione si espande in lacrime divine". Anche, la sua amicizia per Luigi II di Baviera, il re psicopatico, lipemaniaco, dimostra l'affinità elettiva. ${ }^{50}$

Bianchi, Roncoroni e Lombroso, al di là della lettura sul piano clinico, stanno qui esprimendo una posizione largamente condivisa in quel momento. Questa lettura colloca Verdi, con una forzatura che non poteva non infastidire il diretto interessato, ${ }^{51}$ nel quadro del nuovo concetto di stato, nella concezione sociale volontaristica e protorazziale del

50 Cesare Lombroso, Nuovi studi sul Genio, vol. I (Da Colombo a Manzoni), Milano, Sandron 1901 (Appendice).

51 Italo Pizzi (Ricordi verdiani inediti, Torino, Roux e Viarengo, 1901, p. 31) sosteneva che Verdi liquidasse la teoria di Lombroso "ridendo"; un breve commento si trova in Marcello Conati, Interviste e incontri con Verdi, Milano, Il Formichiere, 1980, p. 344-345. 
noi di Turiello: Verdi non è un genio "patologico", "degenerato", affetto da "iperestesia" (sono parole di Lombroso); è invece un lavoratore, un "ingegno" (non un "genio") che produce grazie ad un'attività "progressiva molto più sana e biologica", "sana personificazione della genialità italiana" (Bianchi); parole che non richiedono troppi commenti, nel pieno del sogno imperialista borghese, tanto ambizioso quanto velleitario, di quella nuova classe dirigente.

Tanto il socialista Lombroso, quindi, quanto l'antidemocratico Turiello esprimono l'esigenza di un noi, di un senso di subordinazione all'autorità e all'istituzione statale, sia pur con diversi presupposti e diversi intenti. Se nella realtà Verdi si colloca in una direzione del tutto differente, semmai contraria a questa forma impersonale e antiliberale di statalismo, abbiamo visto che da parte dei positivisti e della nascente psicologia sociale la sua figura è completamente assorbita e funzionalizzata a quella idea di forma-stato. Verdi diventa l'ingegno progressivo, il campione del lavoro, il rappresentante di una identità creata $a d$ boc, non il genio isolato, degenerato, malato, "lipemaniaco". Nel discorso dell'identità nazione, nella costruzione della sintassi nazionalista italica (con una radicale forzatura di ciò che Verdi realmente diceva e rappresentava) tanto il noi di Turiello quanto l'anti-genio di Lombroso contribuiscono a questa immagine del Verdi che "pianse ed amò per tutti".

L'idea di Lombroso ebbe un seguito anche da parte di un altro suo collaboratore, Arturo Morselli (erano colleghi presso l'ateneo di Torino), che scrisse per un periodico decisamente meno specialistico e di larga diffusione, La scena illustrata, un articolo divulgativo intitolato Verdi e la teoria del genio:

La equilibrata personalità di Giuseppe Verdi non può piegarsi, né tanto meno deturparsi per trovar posto nella dottrina psichiatrico-antropologica circa la natura del genio. Fu già detto, ma giova ripeterlo, in un momento come questo nel quale si deve lamentare che la teoria della «degenerazione epilettica» degli uomini superiori, malamente compresa e disgraziatamente applicata da psicologi orecchianti e, quel che è più, da dilettanti di una specie di psichiatria popolare priva d'ogni severo criterio scientifico, si è resa colpevole di inopportune esagerazioni e di sconvenienti escursioni nel campo della letteratura e dell'arte.

[...] Niuna delle stimmate così dette della degenerazione, quali noi clinici vediamo nei nostri pazzi, negli epilettici, negli isterici, nei paranoici, niuno anche dei sintomi che caratterizzano la grande neurosi, esiste in Giuseppe Verdi.

Egli non ha $[\ldots]$ nessuna di quelle anormalità fisiche da cui deve essere 
dedotta la diagnosi di "degenerato". E che si sappia, neppure nella sua esistenza tranquilla e serena egli offre i caratteri dei genii ammalati o anomali: né la impulsività, né la incoscienza, né la suscettibilità meteorica.

[...] Manca in Lui lo scatto impulsivo della creazione "epilettoide"; che anzi tutta la ammirabile opera sua procede gradualmente, per così dire logicamente perfezionandosi, con una corrispondenza stupenda fra gli acquisti lenti e faticosi della tecnica e il prodotto delle novelle associazioni in che risiede la nota distintiva del genio.

In sintesi, il vasto campo del positivismo italiano dedica molto spazio alla comparazione Verdi Wagner, con risultati omogenei seppur con diverse sfumature. Si può sintetizzare questo campo di riflessione nell'opposizione fra il concetto di genio (che parla per se stesso, isolato e degenerato) e quello di ingegno (che esprime un sentire collettivo e nazionalmente caratterizzato, evidente proiezione di un'ideologia del noi); nella lettura di Verdi anti-genio intesa all'interno di una visione politica sostanzialmente conservatrice; nella negazione che l'arte abbia contenuti di verità geniali, metafisici, avanzati e originali, sostituiti da una collocazione di Verdi come voce di un popolo, che contribuisce a comporre in unità una nazione dall'origine disgregata in Arii e Italici. ${ }^{52}$

Naturalmente anche nell'opposto campo dell'idealismo alcuni scrittori e filosofi propongono la comparazione Verdi Wagner, primo fra tutti Antonio Tari. Questi riserva a Verdi un favore sentimentale e ingenuo, una commozione, una partecipazione che nel campo positivista sembra bandita, davanti all'esigenza scientista, statistica e totalizzante. Non mi dilungo su Tari, limitandomi a rimandare agli studi di Francesco Solitario ${ }^{53}$ e a un recente scritto del compianto Elio Matassi. ${ }^{54}$

52 Per queste conclusioni, che traggo dal confronto fra diverse letture d'ambito positivista, sono debitore sia pur mediatamente del capitolo intitolato "L'isola di Lombroso" in Giampiero Moretti, Il genio. Origine, storia, destino, Brescia, Morcelliana, 2011, pp. 153-158.

53 Francesco Solitario, Antonio Tari e la musica, Milano, Prometheus, 2011.

54 Elio Matassi, Giuseppe Martucci, l'estetica di Antonio Tari e la "musica assoluta", in Giuseppe Martucci. Da Capua all'Accademia di Santa Cecilia, a cura di A. Rostagno e P. P. De Martino, Roma, Accademia Nazionale di Santa Cecilia, 2012; dove lo storico della filosofia si impegnava a dimostrare che Tari, pur rimanendo un entusiasta sostenitore di Verdi, non poteva considerarsi un antiwagneriano radicale; e in effetti l'idealismo italiano non pose mai le differenziazioni esclusive dei positivisti. 


\section{MUSICA E SIGNIFICATI}

È ora di passare a brevi esempi musicali tratti dalla tarda fase intellettuale di Verdi; è un tentativo di trovare nel piano musico-drammatico il riflesso del piano socio-culturale e politico fin qui svolto, quell'intreccio di piani che ho programmaticamente accennato in apertura.

Uno degli esempi che mi sembrano più chiari è il finale secondo di Aida, com'è noto costruito su una triplice "variazione" della medesima struttura armonica che sostiene tre melodie diverse, in seguito sovrapposte a contrappunto. Oltre l'identica struttura armonica, le tre "variazioni" condividono anche, grosso modo, le note cardine della melodia; ma in superficie emergono nette differenze di qualità espressiva fra i tre gruppi che cantano:

a) La "variazione" del POPOLO (coro misto, "Gloria all'Egitto, ad Iside") è scritta in piena armonia a 4 parti (più la linea del Re ovviamente autonoma, che re sarebbe altrimenti?); il tessuto accordale armonizza anche la lieve dissonanza del VI grado bemollizzato (Do bemolle, sul quale il coro di prigionieri canta la parola "liberi"): una parte (soprani) canta quella dissonanza in modo da integrarla nell'armonia collettiva

b) La "variazione" che identifica il POTERE DEI SACERDOTI (coro maschile, "Inni leviamo ad Iside") non è in armonia ma in rigidi unisoni e ottave; la sua legge semplicemente è per sé, non viene condivisa per convinzione, non entra in armonia come era nel popolo. E significativamente questo potere astratto e assoluto è collocato nel mezzo, fra collettività popolare (prima) e individui soli e timorosi per la loro debolezza (dopo)

c) Nella "variazione" in cui cantano gli INDIVIDUI (Aida e Radames, "Qual speme omai più restami/D'avverso nume il folgore") tutte le dissonanze sono 'vissute', ossia introiettate, acquisite dentro la loro esile linea melodica accompagnata dall'arpa (strumento consuetamente associato da Verdi alla preghiera).

La collocazione delle tre aree espressive è decisiva, come lo è la dialettica armonia/unisoni/linea solistica con arpa; anzi la 'non-dialettica', poiché questa disposizione delle parti ritrae un tipo di potere cieco e sordo, che anziché unire individuo e collettività si pone in mezzo, dividendoli. Se si passa l'accostamento: questo finale si può leggere come una denuncia, realizzata con mezzi puramente tecnico-compositivi, di una irreparabile contraddizione del principio del liberalismo moderato, 
la interruzione della continuità fra bene collettivo e la salvaguardia dell'individuo di cui ho largamente parlato nelle pagine iniziali.

La conferma di questa potenza che annienta sotto la sua immobile volontà, di questo potere politico e religioso dei sacerdoti, viene dalla successiva pagina, dove la sovrapposizione in contrappunto delle tre linee non porta affatto a un concertato consueto, dominato dalla linea celestiale e redentrice del soprano, dalla voce dell'eterno femmineo come in Rigoletto, Traviata, Ballo in maschera o ancora nel Boccanegra e persino, sebbene provvisoriamente, con Desdemona nel finale terzo. No; il grande sforzo di armonizzare che Verdi fa nella pagina contrappuntistica viene vanificato dall'approdo, dall'esito a cui questo sforzo conduce: tutto finisce nel tema immobile dei sacerdoti (Più mosso), che prelude al ritorno della marcia trionfale. In tal modo i contrasti delle "variazioni" sono risolti dall'intervento d'autorità dei Sacerdoti (potere illiberale e totalitario), il quale affida l'ultimo momento al suo braccio armato, alla sua forza imponente, la forza militare simboleggiata dalla marcia appunto: messo a tacere il popolo, tacitati gli individui, rimane il potere illiberale e il suo braccio armato, la volontà di potenza e la sua espressione sul campo di guerra. L'infrazione dell'armonia non realizzata lascia campo libero alla forza dell'esercito, all'esercizio della volontà di potenza, alla ricerca dello spazio vitale colonialista se vogliamo azzardare una lettura storicistica. È uno spirito aggressivo e del tutto opposto all'umanitarismo mazziniano e all'armonia sociale liberale, i cui pericoli Verdi sente assai precocemente sin dall'anno di Sedan: una svolta storica nella mentalità europea, che lascerà un segno profondo fin nel pieno Novecento.

Diversa concezione del potere si trova invece nel grande duetto Aida-Amonasro nel III atto (Fig. 1). Che Amonasro non sia il potere astratto e gelido, e che pur essendo un feroce guerriero non sia portatore del totalitarismo politico-religioso dei Sacerdoti egizi, è chiaramente manifestato dalla scrittura musicale che Verdi gli riserva. Quando la figlia cede alla ragion di stato e chiede pietà ("Pietà!/ Padre ... a costoro/ schiava non sono/ [...] della mia patria/ degna sarò"), Verdi assegna al padre una delle linee melodiche più nobili e commoventi della partitura

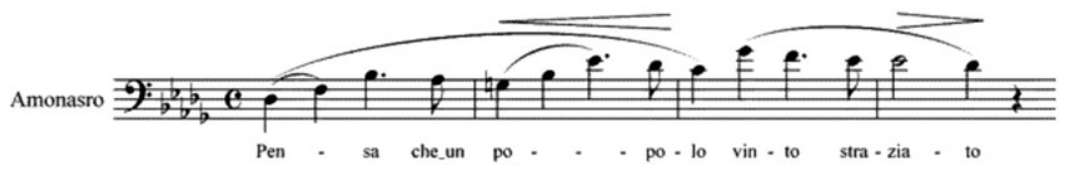

Fig. 1. G. Verdi, Aida, Atto III: Duetto Aida-Amonasro. 
il cui significato viene chiarito dalla quasi identità con la linea dalla preghiera di Leonora nella Forza del destino "Non mi lasciar, soccorrimi, Pietà, Signor, Pietà!” (Fig. 2): $:^{55}$

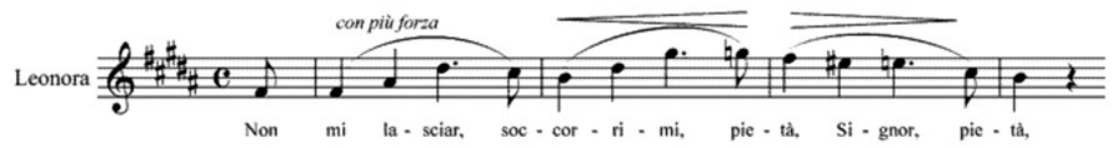

Fig. 2. G. Verdi, La forza del destino, Atto II, scena V.

La ricorrenza dell'unica parola comune ai due momenti, "pietà" appunto, indica il rilievo assegnato al concetto di compassione, che emerge dalla scena di Aida. Compassione per il prossimo, responsabilità verso i propri simili, sacrificio dell'individuo per il bene comune, sono infatti tre fondamenti del liberalismo classico, che accomunano la generazione di Mazzini e quella di Verdi.

$\mathrm{Ma}$ ancor più chiaramente quella continuità fra regnante e suddito, fra potere e sottoposto, fra padre e figlia, fra "governi e governati" per usare il titolo di Turiello, si manifesta nella perfetta continuità con cui la linea di Aida scaturisce dalla cadenza evitata di Amonasro: il Re bemolle conclusivo della linea di Amonasro dalla funzione di tonica maggiore viene trasformato nella settima su una doppia dominante, da cui prende avvio senza soluzione la linea tormentata di Aida, che finisce per cadenzare molto più avanti in Re bemolle minore.

Il senso del dovere e la compassione sono gli elementi psicologico-emotivi che spirano da questa pagina grondante sofferenza in entrambi i personaggi, in una delle scene psicologicamente più violente del teatro verdiano. Da questa pagina emerge anche il rimpianto di Verdi per un tipo di umanità, di cittadino, di persona pronta al sacrificio per il bene comune, educata al rispetto reciproco per il bene collettivo; ossia quello che avrebbe voluto contribuire a realizzare con nume-

55 L'analogia fra le due linee melodiche è ancor più sorprendente e significativa quando si osservi la radicale differenza metrica dei due testi poetici: Leonora canta due settenari (sdrucciolo-piano), mentre Amonasro canta due doppi quinari. Non è casuale quindi che la prosodia di quest'ultimo risulti incerta, quasi forzata (la sillaba accentata della parola popolo è quasi innaturale e inusuale per Verdi). Ciò rafforza l'ipotesi che Verdi abbia ricercato deliberatamente il riferimento intertestuale, forzando la declamazione del verso per ottenere una significazione di livello diverso. 
rosi esempi del suo teatro, ma che la classe politica non aveva secondo lui saputo creare.

Aida è stata letta più d'una volta come opera wagneriana, e Verdi stesso se ne lamentava con un certo distacco ironico:

Dopo 25 anni che io era assente dalla Scala ho ottenuto un fischio dopo il primo atto nella Forza del destino. Dopo l'Aida ciarle infinite: che non era più il Verdi del Ballo (di quel Ballo che fu fischiato la prima volta alla Scala): [...] e che infine era un imitatore di Wagner!!! Bel risultato dopo 35 anni di carriera finire imitatore. ${ }^{56}$

Eppure anche coloro che proiettarono l'infatuazione wagneriana su Aida, come Filippo Filippi, ${ }^{57}$ non davano soltanto un giudizio soggettivo o impressionistico. Queste allusioni al wagnerismo di Aida lasciano invece intravedere qualcosa di più radicato e diffuso nel contesto socio-culturale italiano sin dagli anni Settanta; sono segni di una proiezione della deutsche Bewegung anche su Verdi. E questo rimarrebbe valido anche se, con un esercizio di intelligenza analitica, ci sforzassimo di trovare qualcosa di wagneriano nel "livello neutro", nella grammatica e nella sintassi dell'opera verdiana; certo, qualcosa di wagneriano potremmo anche trovarlo, ma senza alcuna incidenza su quei significati sociali, politici e culturali di cui ho parlato sopra. Su questo piano, l'interferenza fra deutsche Bewegung e produzione artistica di Verdi è del tutto fuorviante, è una lettura storiografica del tutto opposta a quella che qui propongo, senza che questo significhi tornare a fare di Verdi un cocciuto e anacronistico nazionalista, ostinatamente impermeabile alle sollecitazioni che arrivavano dal mondo germanico.

Che poi la lettura di Aida in chiave wagneriana sia tendenziosa e pregiudiziale, nel momento in cui inizia il "movimento tedesco", viene confermato anche da altre considerazioni. Anzitutto abbiamo già visto che, quando Aida arriva a Vienna nel 1875, Hanslick non ci trova proprio nulla di tedesco e men che meno di wagneriano (cfr. nota 41). In

56 Verdi a Giulio Ricordi, 4 aprile 1875 (Abbiati, Verdi, vol. III, pp. 748-49); il 19 marzo1878 il compositore ripete le stesse parole alla Maffei: "Per qual motivo scriverei? [...] Sentirei da capo a dirmi che non ho saputo scrivere, e che son diventato un seguace di Wagner. Bella gloria! Dopo quasi quarant'anni di carriera finire imitatore!" (Julian Budden, Le opere di Verdi, Torino, Edt, 1988, vol. III, pp. 317-318).

57 Cfr. le testimonianze in questo senso riportate da Budden, op. cit. vol. III, p. 48. 
secondo luogo le considerazioni fatte intorno al finale secondo definiscono un'idea politica e una concezione dello stato di Verdi del tutto opposta all'idea di Machtstaat, di centralismo autoritario e statalista caratterizzante la classe dirigente italiana che realizza il nuovo corso della "via prussiana". Quella scena allude a una denuncia della interruzione che il potere politico opera inframmettendosi fra collettività (coro di popolo) e individui (Aida e Radames). È lo stato di diritto che degenera in stato etico ${ }^{58}$ è il potere assoluto e autoreferenziale che separa individuo e collettività: lo stato come distaccato gestore di norme astratte, che annullano gli individui.

Se Wagner come abbiamo visto era recepito come elemento rappresentativo all'interno di quella deutsche Bewegung, qui Verdi si prende un atteggiamento di ferma e decisa opposizione a quel movimento, indicandone i rischi politici; e su questo piano non si può immaginare una più ampia distanza fra Aida e il wagnerismo, ben al di là di ogni possibile considerazione analitica sul livello neutro del materiale musicale. Mai due concezioni della società e dell'individuo sono state tanto lontane quanto il wagnerismo italiano, sintomo della nuova "via prussiana", e il Verdi di Aida erede tardivo del liberalismo idealistico. Per l'esegesi del melodramma verdiano dalla prospettiva socio-politica, ossia per l'interesse conoscitivo che mi sono proposto in questa sede, occorre quindi muoversi sul piano dei grandi sistemi ideali, della grande tradizione speculativa del liberalismo italiano; occorre inserire la produzione verdiana

58 Oggi per "stato etico", dopo i totalitarismi del Novecento, si intende lo stato che impone anche i principi del comportamento personale privato, una forma di dogmatismo quindi. Ma nel medio Ottocento il sintagma aveva altri significati: per Francesco Fiorentino, Camillo De Meis e i due Spaventa la concezione etica dello stato indica una funzione organizzativa, nel quale ogni individualità trova la sua giustificazione: stato etico come organismo in cui sono compresi gli altri organismi particolari (F. Fiorentino, Lo stato moderno. Lettere a Silvio Spaventa, in "Giornale napoletano di filosofia e lettere" II; 1876, vol. III, pp. 485-502, vol. IV pp. 598-611; ora in Gli hegeliani di Napoli e la costruzione dello stato moderno, Roma, Poligrafico dello Stato, 1989, p. 361). Non quindi scissione fra stato come entità astratta e individui; al di fuori dello stato non c'è possibilità per l'individuo; individuo e stato, sono l'espressione della stessa "eticità". Già Garin aveva indicato "la distanza che passava tra il liberalismo degli Spaventa col sottinteso dello stato etico, il 'comunismo' di un Labriola, e la democrazia o il socialismo 'umanistico', e meglio direbbesi umanitario, cui inclinarono non pochi positivisti” (Garin, op. cit., vol. I, pag. 20). 
nella profonda opposizione fra "via prussiana" e liberalismo risorgimentale italiano, che ha attraversato lo scorcio dell'Ottocento. Ecco perché il famigerato verso di d'Annunzio "pianse ed amò per tutti" nasconde, in modo strumentale e surrettizio, una forzatura di carattere ideologico assai comune: il Verdi liberale moderato, che nel suo schierarsi con i conservatori della Destra storica non rinnega mai l'umanitarismo mazziniano della gioventù, viene così affiliato al nuovo nazionalismo italiano emotivo e irrazionale, dove il canto viene del tutto distaccato, nella sua funzione di commozione istintiva irriflessa, dai contenuti sociali e politici del dramma, che sono invece a mio avviso imprescindibili e mai separabili dalla situazione che li ha generati. Si fa insomma di Verdi una specie di posteggiatore napoletano di alto lusso per classi che possono permettersi il biglietto del teatro, o un canzonettista leggero specchio di un congenito sentimentalismo italiano, primitivo e persino un po' babbeo, per i frequentatori di teatri popolari. ${ }^{59}$

Capovolgendo quelle assurde parole di d'Annunzio, quindi, potremmo ripetere che il terreno d'azione di Verdi sia quello dell'impegno sociale attraverso l'arte: non "pianse ed amò", ma "fece prendere coscienza" di alcuni errori politici radicali (o almeno tentò di farlo) attraverso la commozione artistica, intesa come strumento di comunicazione di contenuti che vanno ben al di là del costrutto sonoro neutro. Semmai, allora, non "pianse" ma "pensò" per tutti, e tradusse le idee in termini emotivamente persuasivi. Possiamo quindi chiudere con una sentenza di Foscolo, su cui credo che Verdi avrebbe assentito senza residui: "sdegno il verso che suona e che non crea". ${ }^{60}$

59 La distinzione fra teatri di tradizione, teatri popolari e politeama (con diversa componente sociale dell'uditorio) emerge con tutta la sua distinzione di classe e di cultura proprio nello scorcio dell'Ottocento, accompagnando il processo di massificazione della cultura e la proiezione forzata dell'intenzione populistica sul teatro di Verdi (cfr. Fiamma Nicolodi, Per un confronto della produzione lirica negli anni post-unitari, in Orizzonti musicali itali-europei 1860-1980, Roma, Bulzoni, 1990, pp. 13-42; Carlotta Sorba, Teatri. L'Italia del melodramma nell'età del Risorgimento, Bologna, Il Mulino, 2001, in particolare il Cap. Quarto "L'opera lirica e l'Italia nuova”, pp. 227-264).

60 Ugo Foscolo, Le Grazie, Inno I (Venere), Carme ad Antonio Canova, verso 25, in Poesie di Ugo Foscolo, nuova edizione critica per cura di Giuseppe Chiarini, Livorno, Giusti, 1904, p. 133. 


\section{APPENDICE - CRONOLOGIA SINTETICA}

1864 Convenzione di settembre fra Napoleone III e Minghetti-Nigra; il parlamento si divide in Permanente (gruppo conservatore piemontese, Sella pro Minghetti) e Consorteria (toscani, liguri, con alcuni meridionali e lombardi: liberalismo cavouriano, limitazione dell'intervento statale).

1865 - Spostamento della capitale a Firenze. Elezioni politiche: molti "uomini nuovi" al parlamento (rinnovato per il $47 \%$ ); la "Sinistra giovane" subito trova accordo con la Permanente (prodromo del trasformismo).

- Bismarck sonda la disponibilità del governo La Marmora a fare guerra all'Austria; l'Italia risponde che deve consultare la Francia.

1866 - 8 aprile: il governo italiano firma l'alleanza militare con la Prussia contro l'Austria.

- Terza guerra d'Indipendenza: "guerra falsa" (Cattaneo), "vittoriosa disfatta".

- Crisi monetaria europea.

- 1 maggio: la Banca Nazionale avvia il corso forzoso.

1867 - Febbraio: nasce la Gazzetta di Torino vicina alla Permanente, ma con forte tendenza regionalistica.

- 27 febbraio: a Napoli viene fondata l'associazione Libertà e Giustizia vicino all'Estrema: programma di suffragio universale, senato elettivo, separazione assoluta dei poteri, libertà di stampa, la libertà di culto.

- Nuove elezioni, governo Menabrea in cui prende rilievo la Consorteria.

- Inizio della crisi economica; forti tagli alla spesa pubblica.

- Fallimento del Crédit Mobileir dei Pereira, che appoggiava la Consorteria e il Credito Mobiliare di Bastogi.

- 8 luglio: discorso di De Sanctis, sulla "anomalia" del parlamentarismo italiano: la cronica mancanza di una maggioranza, di due schieramenti netti (da cui verrà il trasformismo); sottolinea il rilievo politico nazionale della questione cattolica e della questione regionalistica (Capone, pp. 192-3).

- Garibaldi in settembre viene condotto e tenuto sotto sorveglianza a Caprera, per impedirgli la presa di Roma.

- 3 novembre: fatti di Mentana, dopo che i Cairoli avevano perso la vita a Villa Glori il 23 ottobre.

1868 - Aprile-maggio: manifestazioni di piazza contro la tassa sul macinato: inizio della "questione sociale".

- 7 luglio, il governo Menabrea vara la tassa sul macinato (in vigore dal 1 gennaio 1869; sarà tolta solo nel 1884 da Cairoli-Depretis).

- Scandalo della Regìa Cointeressata dei tabacchi, forma di rendita garantita con cessione del monopolio a privati (Credito Mobiliare, Bastogi, Duca di Galliera, e gruppo Rotschild).

- Novembre-dicembre: scandalo di corruzione di tre parlamentari della Destra 
Consorteria: Crispi accusa Fambri Brenna e Civinini di essersi fatti comprare dai banchieri della Regìa Cointeressata.

- Fondazione del periodico La Plebe, organo del socialismo evoluzionista italiano (pubblicato fino al 1883): dichiarata convergenza di socialismo e positivismo, a cui non è estranea la "scapigliatura democratica".

1869 - Napoli: prima sezione dell'Internazionale in Italia; più di mille soci.

- Verdi torna alla Scala di Milano dopo 25 anni: La forza del destino.

1869-1870 - inizio delle trattative di Raffaele Rubattino per l'acquisto della baia di Assab (si concluderanno nel 1882): inizio dell'intenzione coloniale.

1870 - 19 luglio: dichiarazione di guerra della Prussia alla Francia; dichiarazione di neutralità italiana.

- inizio del Kulturkampf anti-cattolico e in nome di un liberalismo laicista (ma in realtà autoritario e statalista); almeno fino al 1879.

- Concilio Vaticano; sulla proclamazione del dogma della "infallibilità del Papa", opposizione e rottura con Pio IX da parte dei prelati francesi e tedeschi.

- Parsifal, in pieno Kulturkampf.

- 1 settembre: Débacle francese di Sedan.

- 20 settembre: Porta Pia.

- Novembre: elezioni politiche italiane, in clima di grande incertezza; emerge il "sistema Sella" (Capone, p. 279): onnipotenza dello stato, protezionismo in economia, accentramento amministrativo.

1871 - Aida al Cairo.

- Estate: distacco Bakunin - Mazzini (l'allontanamento fra i due risaliva al 1865).

- Settembre: Congresso dell'Internazionale socialista di Londra: scissione fra marxisti e anarchici.

- Spostamento della capitale a Roma.

- Prima esecuzione italiana di un'opera di Wagner: Lohengrin a Bologna e Firenze.

- Cesare Lombroso, L'uomo bianco e l'uomo di colore. Letture sull'origine e la varietà delle razze umane (Padova, Sacchetto): teoria delle "due razze" italiane, settentrionale-meridionale; "questione meridionale".

1872 - Aida a Milano.

- Tannbäuser a Bologna e Firenze.

- Lohengrin alla Scala, grande polemica con risvolti politici.

- 10 marzo: morte di Mazzini.

- Rimini: primo congresso della Federazione degli anarchici in Italia.

- Rimini: primo congresso del Movimento operaio-socialista.

1873 - 10 luglio: governo Minghetti di Destra.

- Visita di Vittorio Emanuele II a Vienna, inizio dell'avvicinamento all'Austria.

- Alleanza dei tre imperatori Germania, Austria, Russia (a cui Bismarck avreb-

be voluto aggiungere l'Italia per accerchiare la Francia).

- 30 novembre: I Goti di Gobatti, Bologna, Teatro Comunale.

1874 - Febbraio: accordo del governo Minghetti con la "Sinistra giovane" di De Sanctis; inizio del trasformismo. 
- Inizio della "Nuova scuola" economica, sociale e politica: antiliberalismo, statalismo anche in economia, modello germanico di Adolf Wagner e Lujo Brentano, industrialismo, "socialismo della cattedra".

- Luigi Luzzatti, Alessandro Rossi, Robecchi e Scialoja: conclusione dell'inchiesta sull'industria italiana (La Nuova Antologia, XXVII, settembre 1874, pp. 174 192).

- Francesco Ferrara, Il germanesimo economico in Italia (Nuova Antologia, XXVI agosto, ora in Opere complete, a cura F. Caffè, Roma, 1972): ragioni storiche del liberismo in Italia.

- Luigi Luzzatti, L'economia politica e le scuole germaniche (Nuova Antologia, XXVII settembre, 174-192).

- Nuove elezioni: emergono due gruppi distinti sia per provenienza geografica sia per indirizzo economico: gruppo statalista-industrialista settentrionale (Sella), gruppo agrario meridionale (Nicotera).

- Primo Congresso Cattolico a Venezia; fondazione dell'Opera dei Congressi.

1875 - 4-6 gennaio: Congresso degli economisti a Milano (antiliberisti).

- Fondazione della Società Adamo Smith da parte dei liberisti Peruzzi, Ricasoli, Bastogi (destra), Ferrara, Mancini (sinistra); pubblica il periodico L'Economista. - Fondazione della Associazione per il progresso degli studi economici, Pasquale Villari (destra), Toniolo (cattolico), Bertani e Gabriele Rosa (radicali repubblicani), "socialisti della cattedra"; pubblica il periodico Il Giornale degli Economisti.

- 4 luglio: discorso di Nicotera a Salerno, esigenza di una politica meridionalista.

- 1 agosto: riunione di Depretis a Torino conto Nicotera; anti-meridionalismo piemontese.

- 5 agosto: L'Opinione pubblica una Corrispondenza da Torino: in parlamento esistono "due razze", la razza nordica prevale e siede a destra [...] la razza meridionale prevale e siede a sinistra"; auspica che la divisione finisca.

- 10 ottobre: Depretis, discorso di Stradella (contro Nicotera): questione ecclesiastica, istruzione primaria obbligatoria, decentramento, allargamento del suffragio, il pareggio di bilancio "non è tutto".

- Verdi: tournèe a Parigi, Londra e Vienna con il Requiem (in progetto, irrealizzato, era anche Berlino).

1876 - Pareggio di bilancio raggiunto dal governo Minghetti.

- 25 marzo: "Rivoluzione parlamentare", Depretis e la sinistra storica al governo; in realtà un semplice avvicendamento di maggioranze.

- Novembre: elezioni con suffragio allargato; declino della destra.

- De Sanctis, svolta al realismo (Il principio del realismo, in "La Nuova Antologia” gennaio 1876, ora in Saggi critici, a cura di L. Russo, Bari, Laterza, 1957, III/219-235).

- Lombroso, L'uomo delinquente studiato in rapporto all'antropologia, alla medicina legale ed alle discipline carcerarie (Milano, Hoepli).

- 13 novembre, Verdi a Piroli: "Chi sa quale piega vanno a prendere le cose nostre in avvenire! Non è il colore che mi fa paura, ma temo gli Apostoli come diceva Giusti. Temo la poca capacità, la violenza, l'intolleranza di questo partito 
[Sinistra storica], e temo soprattutto il braccio debole e vacillante di De Pretis. Chi sa che un giorno e Correnti e i Toscani non abbiano a pentirsi di aver cooperato a questo risultato" (contro il trasformismo).

- 15 dicembre, Verdi a Piroli: "Da che sono qui [a S. Agata] leggo molto i giornali, e di tutti i colori: ne capisco meno di prima ... cioè no, capisco che le cose vanno o possono andare più tardi male assai".

- Fondazione del Correre della sera.

1877 - Guerra Russo-Turca; si riapre la questione balcanica. Dichiarazione di neutralità dell'Italia.

- "Terzo partito" nato dalla convergenza di Sella, Cairoli e De Sanctis.

- 12 marzo, Piroli a Verdi: "La 'nuova scuola', o come già la chiamano la nuova consorteria progressista si compone in gran parte di gente ignorante"; Sella prega Verdi di andare a Colonia per "motivi anche politicamente" fondati.

- 27 marzo, Verdi a Piroli: si definisce "il più gran pessimista che esista [...]. Non capisco quello che vi disse Sella. Questi Festivals in Germania hanno una grande importanza artistica ed io non cerco di più.

- Maggio, Verdi a Colonia.

- Estate: incontro Crispi-Bismarck a Gastein/Friedrichsruhe; Bismarck non accoglie le richieste di alleanza anti-austriaca e propone un patto a tre.

- Giugno-novembre: su Il Diritto De Sanctis pubblica una serie di articoli contro l'"affarismo" di Nicotera.

- 6 luglio: nuovo trattato commerciale Italia-Francia (approvato dal Parlamento italiano nel 1878); Depretis rimane ostile al protezionismo, più favorevole Sella.

- 15 luglio Legge Coppino sulla scuola elementare (Duggan 315).

1878 - 9 gennaio: morte di Vittorio Emanuele II.

- 7 febbraio: morte di Pio IX.

- 12 marzo, Verdi a Piroli: "la miseria è molta [...] si tratta di fame!!!", crisi soprattutto nelle campagne.

- 26 marzo: nuovo governo Cairoli; liberalismo e imparzialità secondo lo spirito dello Statuto.

- 3 aprile: problemi per il nuovo trattato commerciale con la Francia.

- 15 aprile: nuova tariffa doganale protezionista, contro le importazioni dalla Francia.

- 13 giugno-13 luglio: Congresso di Berlino, conclusione della guerra RussoTurca (centralità prussiana negli equilibri internazionali); per l'Italia partecipa il ministro degli esteri Luigi Corti ("politica delle mani nette").

- luglio: partecipazione dell'Orchestra della Scala, diretta da Franco Faccio, all'Esposizione Universale di Parigi con esecuzioni sinfoniche; Verdi è contrario. - Leone Carpi: L'Italia vivente. Aristocrazia di nascita e del denaro, borghesia, clero, burocrazie. Studi sociali (Milano, Vallardi): la borghesia è "il nerbo della nazione", con un capovolgimento del liberalismo moderato in utilitarismo individualista, conseguenza della teoria del self-help di Smiles.

- 17 novembre, Napoli: attentato dell'anarchico Giovanni Passannante a Umberto I (fallito). 
- Roberto Ardigò, La morale dei positivisti, in Rivista repubblicana (ristampato separatamente l'anno dopo): fondamento dell' “etica democratica”, dimostrazione del condizionamento ambientale.

1879 - Ascesa parlamentare dei radicali: Felice Cavallotti diviene la voce degli operai. - Aprile, Roma: Assemblea delle forze democratiche, presidente Garibaldi; partecipano Carducci, Saffi, Bertani, Mario, Cavallotti, Bovio, Imbriani ecc.; nasce la Lega della democrazia con programma liberale avanzato e anticlericale.

- 11 giugno, Verdi a Piroli: ignorando la crisi agricola "il Governo [Cairoli] pensa ad aumentare le imposte, a far Strade Ferrate non di prima necessità col pretesto di dare lavoro alla gente? È veramente uno scherzo" (Carteggi, III/140).

1880 - Febbraio: Verdi a Parigi per dirigere Aida.

- Aprile-maggio: Depretis ministro dell'Interno scioglie le Camere e indice nuove elezioni; la destra riconquista molti seggi; frattura nella sinistra fra Depretis-Cairoli e Crispi-Nicotera (sinistra meridionale)

- Fase acuta della crisi agraria, che durerà fino alla metà dei Novanta ed emargina ulteriormente il Meridione.

- Novembre: il partito radicale si avvicina all'Estrema; nuova Sinistra.

1881 - Roma: i radicali convocano il "Comizio dei comizi".

- 11 maggio "schiaffo di Tunisi": occupazione francese di Tunisi, che blocca imprese e investimenti italiani già stanziati. Dimissioni del governo Cairoli. Paura italiana di rimanere isolata. Crispi sceglie una strategia di attivismo pragmatista, nazionalismo avventuroso, irrazionale, impulsivo: si afferma la politica di potenza. - 30 aprile: Andrea Costa fonda il Partito Socialista Rivoluzionario Italiano e inizia a Imola la pubblicazione del settimanale Avanti!.

- Agosto: primo congresso del Partito Socialista di Romagna (clandestino): programma per un partito nazionale, rivoluzione violenta, dittatura delle classi lavoratrici.

- Fondazione a Torino della Rivista di Filosofia scientifica, redattore Arturo Morselli (coll. di Lombroso).

- Fondazione della Navigazione Generale Italiana (Florio, Rubattino, Piaggio), favorita dal protezionismo.

- 25-26 settembre: nasce a Milano la Confederazione operaia lombarda; condanna della violenza per mantenere la lotta di classe all'interno della legalità.

- Milano, La Scala: Simon Boccanegra (II versione).

1882 - 10 marzo: il governo italiano acquista la baia di Assab in Eritrea, riconosciuta "colonia".

- 17 maggio, Milano: Fondazione del Partito Operaio italiano.

- 20 maggio: stipula della Triplice Alleanza.

- Diffusione del nuovo principio del Machtstaat: potenza militare, potenza industriale, aumento demografico, divisione aree forti (industrializzate) e deboli (agricole), "Europa interna" ed "Europa esterna", potenziamento delle comunicazioni ferroviarie, capitalismo legato alla finanza, protezionismo, nazionalizzazione delle masse.

- 2 giugno: morte di Garibaldi. 
- Riforma elettorale: la base elettorale sale a 2 milioni (quasi triplicata); limite del censo abbassato da 40 a 19,80 lire, limite di istruzione dalla $4^{\text {a }}$ alla $2^{\text {a }}$ elementare, inclusione nell'elettorato della classe operaia (settentrionale), scrutinio di lista, apertura dello Stato ai ceti medi urbani.

- Divisione in "due Italie" dal punto di vista dei diritti politici: 35,3\% dell'elettorato era in Piemonte, Liguria e Lombardia; solo il 9,2\% in Calabria, Puglie e Lucania.

- Ottobre: nelle elezioni generali i radicali e i socialisti si presentano insieme e ottengono 40 deputati.

- Andrea Costa entra in Parlamento, primo deputato socialista eletto.

- Diminuiscono in parlamento i proprietari terrieri, aumentano gli avvocati ("nuovi notabili"); prima grande vittoria dell'Estrema, che rappresenta la classe operaia in parlamento.

- 20 dicembre: impiccagione dell'irredentista Guglielmo Oberdan per l'attentato a Francesco Giuseppe.

- Pasquale Turiello, Governo e governati in Italia (Bologna, Zanichelli).

- Depretis apre a Minghetti e a Sella, e si forma il Partito Costituzionale, o Ministeriale, un grande centro per contrastare l'ascesa dell'Estrema radicale che ora conta Zanardelli, Nicotera e Crispi.

- Movimento di scioperi operai-contadini "La Boje” nel mantovano (fino al 1885).

1883 - Inizia la fase più complessa del trasformismo.

- 25 novembre, Napoli: viene varata a Napoli la "Pentarchia" (Crispi, Cairoli, Zanardelli, Baccarini, Nicotera): suo organo di stampa è il periodico La Tribuna, pubblicato a Roma, direttore Luigi Roux.

1884 - Terni: fondazione del maggiore stabilimento siderurgico nazionale, la Società Altiforni Fonderie e Acciaierie, ancora sostenuta dallo stato (inizio produzione nel 1886).

- Aumento progressivo dell'antiparlamentarismo sia di destra che di sinistra.

- Gaetano Mosca, Teoria dei governi.

- Fondazione della Società di Mutuo soccorso (Mantova) e dell'Associazione dei lavoratori italiani (Cremona); primi grandi scioperi.

- Conquista di Massaua; proteste della Destra per costi militari eccessivi; proteste della Estrema Sinistra: Andrea Costa: "Né un uomo né un soldo".

1884-85-nuove convenzioni ferroviarie che assegnano la gestione della rete a tre società: Mediterranea, Adriatica (ex-Meridionali), Sicula; il capitale è per la maggior parte tedesco.

- Avvio del capitalismo industriale italiano fortemente sostenuto dallo stato.

1885 - La Francia adotta misure protezionistiche su grano e bestiame sfavorevoli all'Italia.

- Terminano gli scioperi a oltranza nel mantovano ("La Boje"); seguono arresti e processi agli scioperanti, fra gli avvocati della difesa è Enrico Ferri.

- Giuseppe Sergi, Per l'educazione del carattere. Pagine di psicologia sociale e consigli direttivi (Torino, Camilla e Bertolero): italiani divisi in due razze, "Arii" e "Italici". 
- 1885 pubblicazione della Relazione finale dell'Inchiesta Agraria (iniziata nel maggio 1877) condotta da Stefano Jacini e Agostino Bertani: indice dell'impossibilità per l'Italia di proseguire la via "agricolturista".

- Fogazzaro, Daniele Cortis prototipo del "romanzo parlamentare" o "romanzo antiromano".

1886 - L'Italia denuncia il precedente trattato commerciale con la Francia stipulato nel del 1878 da Depretis.

1887 - Aumento del dazio doganale sul frumento, prima forma di protezionismo.

- Gennaio: disfatta di Dogali; morte di Depretis; primo governo Crispi.

- Rinnovo della Triplice Alleanza, più favorevole all'Italia; crescono le preoccupazioni di scontro con la Francia nel Mediterraneo.

- Otello alla Scala.

1888 - Gennaio, nuova tariffa doganale ostile alla Francia; tentativo di stipulare un nuovo trattato commerciale, subito interrotto (febbraio); inizia la "Guerra doganale" Italia-Francia.

- Appoggio crescente del governo Crispi all'industria: inizia l'emigrazione dal Mezzogiorno.

- Bologna: prima italiana del Tristan und Isolde.

1889 - Lettera di Verdi a Piroli, 10 febbraio 1889 (Carteggi IV/190-191): "Dalla mia finestra [a Genova] vedo tutti i giorni un Bastimento, e qualche volta due carichi almeno di mille emigranti ciascuno! Miseria e fame! Vedo nelle campagne proprietari di qualche anno fa ridotti ora a contadini, giornalieri, od emigranti (miseria e fame). [...] E come si potrà andare avanti? Non saranno mica le nostre industrie che ci salveranno dalla ruina! Voi direte che sono un pessimista!”.

- Fase acuta della Grande Depressione.

- Trattato di Uccialli fra Etiopia e Italia.

1891 - Nuovi investimenti del governo Crispi per spese militari: aumento del deficit statale; Crispi, accusato di spendere troppo, accusa la Destra di "politica servile verso lo straniero" perché, prima del 1876, non aveva mai investito sull'esercito (Mammarella-Cacace, La politica estera dell'Italia, Roma-Bari, Laterza, 2006, p. 39).

- Febbraio: crisi del governo Crispi per deficit eccessivo.

1893 - Battaglia di Agordat, colonia eritrea italiana.

- Scandalo della Banca Romana.

- Inizio della guerra italo-etiopica.

- Falstaff alla Scala.

- Manon Lescaut al Teatro Regio di Torino.

1896 - Grave sconfitta di Adua. Proteste di piazza in Italia; grave crisi economica e sociale, forte aumento delle tasse.

- Scioperi, manifestazioni anti-governative. Di Rudinì ordina la chiusura di circoli socialisti e cattolici popolari.

- Lievi segni di distensione verso la Francia.

1898 - 7 maggio, Milano: dopo ulteriori manifestazioni anti-governative, l'esercito cannoneggia la folla facendo un numero imprecisato di morti; "sospensione di ogni libertà, ogni guarentigia costituzionale” (Torelli-Viollier). 
- Giuseppe Sergi, Arii e Italici. Attorno all'Italia preistorica (Torino, Bocca): anarchia come carattere naturale degli Italici, ordine armonico come carattere naturale degli Arii.

1900 - Giuseppe Sergi, La decadenza delle nazioni latine (Torino, Bocca). 